\title{
Marine Oil Slick Detection Using Improved Polarimetric Feature Parameters Based on Polarimetric Synthetic Aperture Radar Data
}

\author{
Guannan Li ${ }^{1,2}$, Ying $\mathrm{Li}^{1,2, *}$, Yongchao Hou ${ }^{1,2} \mathbb{D}$, Xiang Wang ${ }^{3}$ and Lin Wang ${ }^{3}$ \\ 1 Navigation College, Dalian Maritime University, Dalian 116026, China; lgn@dlmu.edu.cn (G.L.); \\ hyc@dlmu.edu.cn (Y.H.) \\ 2 Environmental Information Institute, Dalian Maritime University, Dalian 116026, China \\ 3 National Marine Environmental Monitoring Center, Dalian 116026, China; xwang@nmemc.org.cn (X.W.); \\ lwang@nmemc.org.cn (L.W.) \\ * Correspondence: yldmu@dlmu.edu.cn; Tel.: +86-411-8472-6829
}

Citation: Li, G.; Li, Y.; Hou, Y.; Wang, X.; Wang, L. Marine Oil Slick Detection Using Improved

Polarimetric Feature Parameters Based on Polarimetric Synthetic Aperture Radar Data. Remote Sens. 2021, 13, 1607. https://doi.org/ $10.3390 /$ rs13091607

Academic Editor: Francesco Bignami

Received: 15 March 2021

Accepted: 16 April 2021

Published: 21 April 2021

Publisher's Note: MDPI stays neutral with regard to jurisdictional claims in published maps and institutional affiliations.

Copyright: (c) 2021 by the authors. Licensee MDPI, Basel, Switzerland. This article is an open access article distributed under the terms and conditions of the Creative Commons Attribution (CC BY) license (https:// creativecommons.org/licenses/by/ $4.0 /)$.

\begin{abstract}
Marine oil spill detection is vital for strengthening the emergency commands of oil spill accidents and repairing the marine environment after a disaster. Polarimetric Synthetic Aperture Radar (Pol-SAR) can obtain abundant information of the targets by measuring their complex scattering matrices, which is conducive to analyze and interpret the scattering mechanism of oil slicks, look-alikes, and seawater and realize the extraction and detection of oil slicks. The polarimetric features of quad-pol SAR have now been extended to oil spill detection. Inspired by this advancement, we proposed a set of improved polarimetric feature combination based on polarimetric scattering entropy $H$ and the improved anisotropy $A_{12}-H_{-} A_{12}$. The objective of this study was to improve the distinguishability between oil slicks, look-alikes, and background seawater. First, the oil spill detection capability of the $H_{-} A_{12}$ combination was observed to be superior than that obtained using the traditional $H \_A$ combination; therefore, it can be adopted as an alternate oil spill detection strategy to the latter. Second, $H\left(1-A_{12}\right)$ combination can enhance the scattering randomness of the oil spill target, which outperformed the remaining types of polarimetric feature parameters in different oil spill scenarios, including in respect to the relative thickness information of oil slicks, oil slicks and look-alikes, and different types of oil slicks. The evaluations and comparisons showed that the proposed polarimetric features can indicate the oil slick information and effectively suppress the sea clutter and look-alike information.
\end{abstract}

Keywords: oil spill detection; polarimetric synthetic aperture radar; improved polarimetric feature parameters; different oil spill scenarios

\section{Introduction}

The oceans play a crucial role on the global ecosystem because they affect the global ecological balance and provide resources and energy [1,2]. Given the rapid development of the global maritime industry, oil spill accidents resulting from collisions involving oil-carrying ships, illegal sewage discharge, and pipeline ruptures occurring frequently, increasing the risk of oil spills in the marine traffic environment [1,2]. Marine oil spills present a global phenomenon, whether in open water or offshore, and receive extensive public attention as a major environmental pollution problem [1-3]. Hence, their rapid and effective detection is of vital significance for strengthening the emergency response involved in maritime traffic safety, search and rescue, and repairing the marine environment after disasters [1,4-6].

The traditional direct measurement or buoy survey has high costs, small coverage, and is greatly affected by weather factors; as such, buoy surveys frequently fail to achieve timely large area detection of oil spills. Synthetic aperture radar (SAR) remote sensing technology 
breaks the limitations of traditional monitoring methods, and has thus been extensively used in the field of detecting marine oil spills because of its all-day and all-weather microwave imaging capability [1,6-9]. The continuous development and improvement of SAR systems has gradually expanded such approaches from single polarimetric to PolSAR $[10,11]$. The Pol-SAR system can obtain the scattering echo signal of a target based on the linear transmit-receive polarization channel combination [12]. The scattering echo information of a target is influenced by its physical characteristics such as material properties, geometric structure, and roughness [6,8]. Therefore, the Pol-SAR system obtains sufficient scattering information and measures the scattering matrix of the target through a record of amplitude and phase data measured at different polarization channels combination $[4,6]$.

The extraction of polarimetric features is the primary process during the analysis and detection of marine oil slicks with polarimetric SAR images, and the core link to identify and classify oil spills based on the differences between the scattering properties of oil slicks, lookalikes, and seawater [13]. Cloude proposed a method utilizing the polarization coherent matrix to decompose and then explore the scattering mechanism in 1985 because the characteristic vector obtained after decomposition could indicate the scattering mechanism of the target object $[10,14]$. Singha assessed the ability to discriminate between oil slicks and look-alikes under ten polarimetric feature spaces, demonstrating the effectiveness of the surface scattering fraction, scattering diversity, and span in oil spill detection [15]. Skrunes et al. proposed a modified anisotropy $A_{12}$ calculated based on the two largest eigenvalues of the scatter matrix; this differs from conventional anisotropy $A$, which is calculated using the two smallest eigenvalues [6]. Zhang modified the conformity coefficient $\mu$ for application to ocean oil slick detection, introducing a land classification for discriminating surface scattering characterized by double-bounce or volume scattering. The results demonstrated that the conformity coefficient is an effective logical classifier for oil spill detection under low to moderate wind conditions [12]. Yang et al. proposed the similarity parameter for measuring the similarity between two scattering matrices [16] and Tong introduced and improved the self-similarity parameter $r r r_{s}$ to enhance discrimination between oil slicks and look-alikes; their analytical results demonstrated that the self-similarity parameter is superior to other three types of polarimetric features parameters [13]. Furthermore, specific combinations of polarimetric features have been applied in the study of oil spill detection. Liu et al. proposed a new combined parameter, $F$, composed of entropy $H$, anisotropy $A$, mean scattering angle $\alpha$, and the magnitude of the correlation coefficient $\rho_{\mathrm{co}}$; their results performed well in detecting oil slicks [17]. Schuler analyzed the properties of oil slicks under combinations of $H$ and $A$, thereby establishing the slick mapping algorithm [18]. Certain studies divided polarimetric features into different categories as per their principles and definitions [13,15]. In this study, these are further refined into four categories as described in Table 1, including backscattered energy types, backscattering mechanism types, the correlation between polarization channels types, and a combination of polarimetric features.

Table 1. Categories and definitions of polarimetric features.

\begin{tabular}{ccc}
\hline Categories & Type & Definition \\
\hline I & Backscattered energy & $\begin{array}{c}\text { The backscattering intensity difference } \\
\text { between oil slick and sea }\end{array}$ \\
\hline II & Scattering mechanism & $\begin{array}{c}\text { The dominating scattering mechanism } \\
\text { difference between oil and slick } \\
\text { (non-Bragg) and seawater (Bragg) }\end{array}$ \\
\hline III & Correlation between polarization & $\begin{array}{c}\text { The difference of channel correlation } \\
\text { channels }\end{array}$ \\
between oil slick (lower) and sea (higher)
\end{tabular}


In summary, the construction and extraction of effective polarimetric feature parameters can enhance the contrast between oil slicks, look-alikes, and seawater, and can expand the polarimetric feature space to facilitate the effective identification and classification of oil slicks. Multiple studies have explored different types of polarimetric feature parameters for oil spill mapping and detection under different oil spill scenes and data sources; however, the extraction of high-quality polarimetric features parameters remains a crucial research objective of oil spill detection research. In particular, it is necessary to highlight oil slick signals and suppress false alarm signals on the basis of expanding polarimetric feature space. Thus, in this study, we construct a new combination feature parameter by analyzing the differences between the scattering mechanisms of oil slicks and seawater, thereby improving the ability to distinguish oil slick, look-alikes, and seawater under different oil spill scenes. We take inspiration for our approach from the research described above concerning the extraction of polarimetric features, as well as the improvement and expansion of research into oil spill detections to form the background and framework for the present study. Compared with previous studies, this study contributes two novel aspects. First, a new set of combination polarimetric features triggered by the traditional combination of polarimetric features were proposed and can be used as an alternate strategy to the latter in oil spill detection. Second, determining the optimal combined polarimetric features $H\left(1-A_{12}\right)$ further expands the polarimetric feature space in oil spill detection applications. The effectiveness and robustness of our proposed features is illustrated through comparison with other categories of polarimetric features under different oil spill scenarios.

The rest of this study is organized as follows. Section 2 introduces the experimental data and sample data acquisition. The theoretical basis and methodology framework of polarimetric features in oil spill detection are introduced in Section 3. The qualitative and quantitative analysis and comparison of oil spill detection ability with different polarimetric features are introduced in Section 4, and Sections 5 and 6 present the major discussion and conclusions of this study.

\section{Experimental Design and Dataset}

\subsection{Experimental Design and Dataset Overview}

The oil pollution of the ocean surface is a major environmental problem. When oil spill accidents occur, by understanding the oil spill distribution information, particularly, the relative thickness of the oil slick, properties, and oil type, it has been possible to assist in the emergency decision-making, cleaning up, and recovery [19]. According to the experiments and analysis results demonstrated by [20], the damping ratio of mineral oil increases as the thickness of the oil slick layer increases. Hence, the reduction in the effective dielectric constant from the relatively thick oil slick can lead to lower in backscattered energy (backscattered intensity) than a thinner oil slick. Moreover, under action from wind, an oil slick on the leeward side with a visible dark edge is relatively thicker than that on the windward side produced a "feathered" oil areas [20-22]. It should be noted that the relative thickness of the oil slick in this paper is a qualitative study introduced from the previous study [19-22], and the oil slick types mainly include mineral and plant oil, which can provide reference and foundation for the future quantitative study of oil slick thickness and oil types.

In this study, we compared and focused on the oil spill identification ability between different types of polarimetric features with three RADARSAT-2 datasets, corresponding to three different oil spill scenarios, which were to avoid the interference on the results brought by different sensors, and used to illustrate the effectiveness and robustness of the proposed method. These datasets include information regarding the relative thickness of the oil slick, oil slick and look-alikes, as well as data for different types of oil slick. RADARSAT-2 provides operation imaging, thereby allowing access to data with a reduced chance of conflicts, including, e.g., Fine, Wide, Standard, Spotlight, and ScanSAR, and supports linear single-, dual-, and quad-polarization options [4,11,12]. The capabilities of RADARSAT-2 quad-polarization data, incorporating extremely low noise-floor and 
cross-talk corrections for different channels, makes it a remarkable system for oil spill detection research [11,12]. In the following, we describe polarimetric SAR data products, formation mechanisms for different oil spill scenarios, field research, and sample data acquisition in detail. Figures 1 and 2 show data visualization images and sample areas of different oil slicks, together with the corresponding formation mechanisms of oil slicks under different scenarios in the experiment.

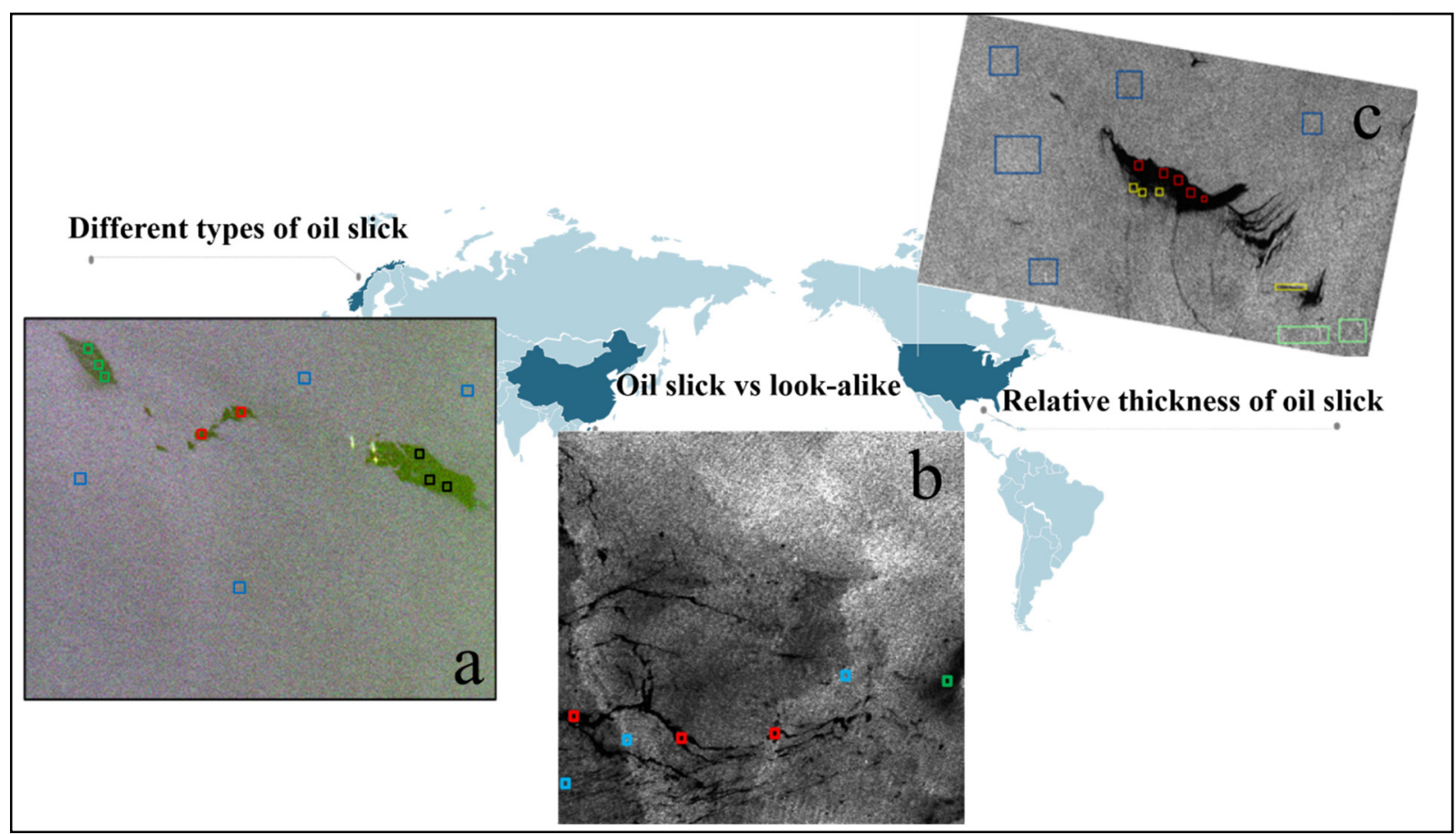

Figure 1. Locations of marine oil spill images obtained by RADARSAT-2 and utilized in this study: (a) Case 1; (b) Case 2; (c) Case 3 .

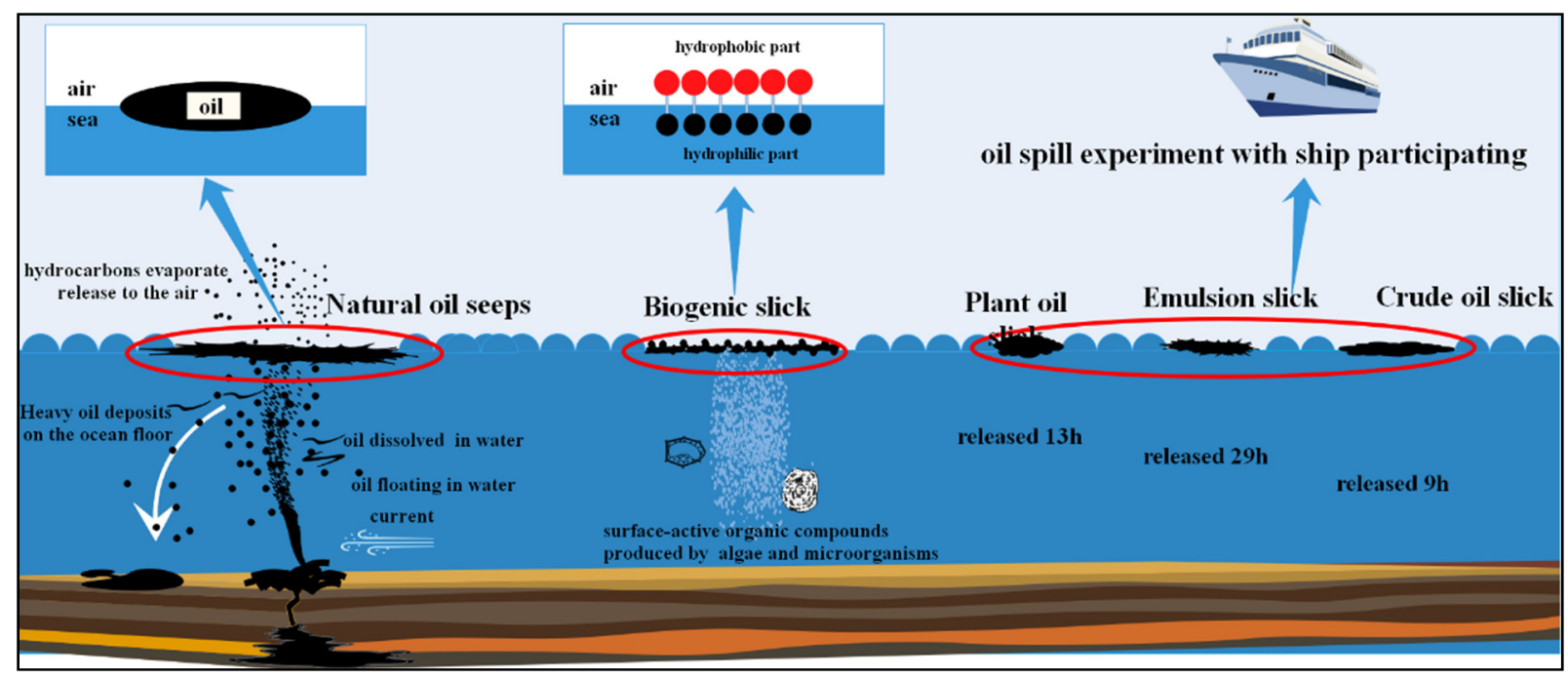

Figure 2. Schematic of different types of oil slicks formed in the marine environment. 


\subsection{Dataset}

Herein, we examined three C-band single look complex (SLC) fine quad-polarization RADARSAT-2 images. Table 2 shows the SAR data for different oil spill scenes utilized in this study.

Table 2. Properties and descriptions of the three SAR scenes used in this study.

\begin{tabular}{cccc}
\hline Case/Sensor & Case 1 (RADARSAT-2) & Case 2 (RADARSAT-2) & Case 3 (RADARSAT-2) \\
\hline Date & $8 \mathrm{May} 2010$ & 18 September 2009 & $8 \mathrm{June} 2011$ \\
Time & $12: 01$ a.m.(UTC) & $10: 50$ a.m.(UTC) & $17: 27 \mathrm{a} . \mathrm{m} .(\mathrm{UTC})$ \\
Region & The Gulf of Mexico & The South China Sea & The North Sea \\
Mode/Product & $\left(26^{\circ} 48^{\prime} \mathrm{N}, 92^{\circ} 02^{\prime} \mathrm{W}\right)$ & $\left(18^{\circ} 06^{\prime} \mathrm{N}, 109^{\circ} 24^{\prime} \mathrm{E}\right)$ & $\left(59^{\circ} 59^{\prime} \mathrm{N}, 2^{\circ} 27^{\prime} \mathrm{E}\right)$ \\
Polarization & Fine Quad-Pol SLC & Fine Quad-Pol SLC & Fine Quad-Pol SLC \\
Frequency & $\mathrm{HH}, \mathrm{HV}, \mathrm{VH}, \mathrm{VV}$ & $\mathrm{HH}, \mathrm{HV}, \mathrm{VH}, \mathrm{VV}$ & $\mathrm{HH}, \mathrm{HV}, \mathrm{VH}, \mathrm{VV}$ \\
Incidence angle & $\mathrm{C}-\mathrm{band}(5.405 \mathrm{GHz})$ & $\mathrm{C}-\mathrm{band}(5.405 \mathrm{GHz})$ & $34.5^{\circ}-36.1^{\circ}$ \\
Wind speed & $41.9^{\circ}-43.4^{\circ}$ & $32.4^{\circ}-33.2^{\circ}$ & $1.6-3.3 \mathrm{~m} / \mathrm{s}$ \\
Slicks present & $6.5 \mathrm{~m} / \mathrm{s}$ & $10 \mathrm{~m} / \mathrm{s}$ & Crude/Emulsion $/ \mathrm{plant}$ \\
Information & Natural oil seeps & Biogenic slick & Difference between types of \\
& Relative thickness of oil slick & slick vs look-alikes & oil slick \\
\hline
\end{tabular}

\subsubsection{Dataset 1-Relative Thickness of Oil Slick}

The RADARSAT-2 image in Case 1 was acquired over well-known oil slicks in the Gulf of Mexico at 12:01 UTC on 8 May 2010, to analyze and interpret the relative thickness difference of the oil slick. The visible dark slicks have been interpreted as natural oil seeps that frequently occur in this area $[2,11,12,23]$. Wind field information was obtained from the nearest buoy ( $\# 42047$ at $27^{\circ} 53^{\prime} 48^{\prime \prime} \mathrm{N} 93^{\circ} 35^{\prime} 50^{\prime \prime} \mathrm{W}$ ) of the National Oceanic and Atmospheric Administration National Data Buoy Center, $\sim 100 \mathrm{~km}$ north of the SAR image location. The wind speed obtained via SAR image acquisition was $6.5 \mathrm{~m} / \mathrm{s}$ in an approximately southern direction $\left(167^{\circ}\right)[11,12]$. Studies based on experiments and analysis demonstrated that, under wind action, an oil slick with a visible dark edge line on the leeward side is relatively thicker than that on the windward side, which usually produces a "plume" oil slick [20-22]. Consequently, relatively thick oil samples were selected at the northern leeward side region and is shown with red box, while thin oil samples were selected in the feathered tail areas of the southern windward side shown with yellow box, seawater samples were selected from the region shown with blue box, and the bright-dark strips caused by ocean waves were selected as look-alikes from the region shown with green box, as shown in Figure 1.

\subsubsection{Dataset 2-Oil Slick and Look-Alikes}

The RADARSAT- 2 image in Case 2 was acquired over a region near a popular fishing locality in the South China Sea at 10:50 UTC on 18 September 2009, to analyze differences between oil slicks and look-alikes. Visible dark slicks have been interpreted as biogenic slicks near the fishing region, whereas the look-alikes are caused by atmospheric fronts [24]. Note that although biogenic slicks are different from traditional mineral oil slicks, studies of their scattering characteristics can be used as a basis and reference for future research Therefore, oil slick samples were selected from the biogenic slicks and shown with a red box, whereas look-alike samples were selected in the atmospheric front region shown with a green box, and seawater samples were selected from region shown with a blue box, as shown in Figure 1.

\subsubsection{Dataset 3-Different Types of Oil Slick}

The RADARSAT-2 image in Case 3 was acquired over oil slicks released during a manufactured oil on water experiment conducted by the Norwegian Clean Seas Association for Operating Companies (NOFO) in the North Sea at 17:27 UTC on 8 June 2011, and was used to analyze and compare differences amongst crude oil, emulsion oil, and plant oil [6]. 
The oil slick from left to right is plant oil, emulsion and crude oil, respectively. Note that $0.4 \mathrm{~m}^{3}$ of Radiagreen ebo plant oil, $1 \mathrm{~m}^{3}$ of emulsion of Oseberg blend crude oil mixed with $5 \%$ IFO380, and $30 \mathrm{~m}^{3}$ of evaporated Balder crude oil were released 13, 29, and $9 \mathrm{~h}$ before SAR image acquisition $[6,13]$. The black, red, green, and blue boxes indicate the regions of sampling of crude oil slick, emulsion oil slick sample region, plant oil slick sample region, and the sea water, respectively.

\section{Methodology}

Herein, we propose new polarimetric features for oil spill detection and classification; Figure 3 shows the flow chart of the proposed method. This method is divided into two main steps: in the first step, a combination of new polarimetric features is extracted and compared with traditional combined polarimetric features. This includes scattering mechanism analysis, polarimetric feature construction, qualitative comparison, and quantitative comparison. In the second step, the optimal polarimetric features $H\left(1-A_{12}\right)$ are further compared with other categories of polarimetric features using three quantitative evaluation measures under different oil spill scenarios.

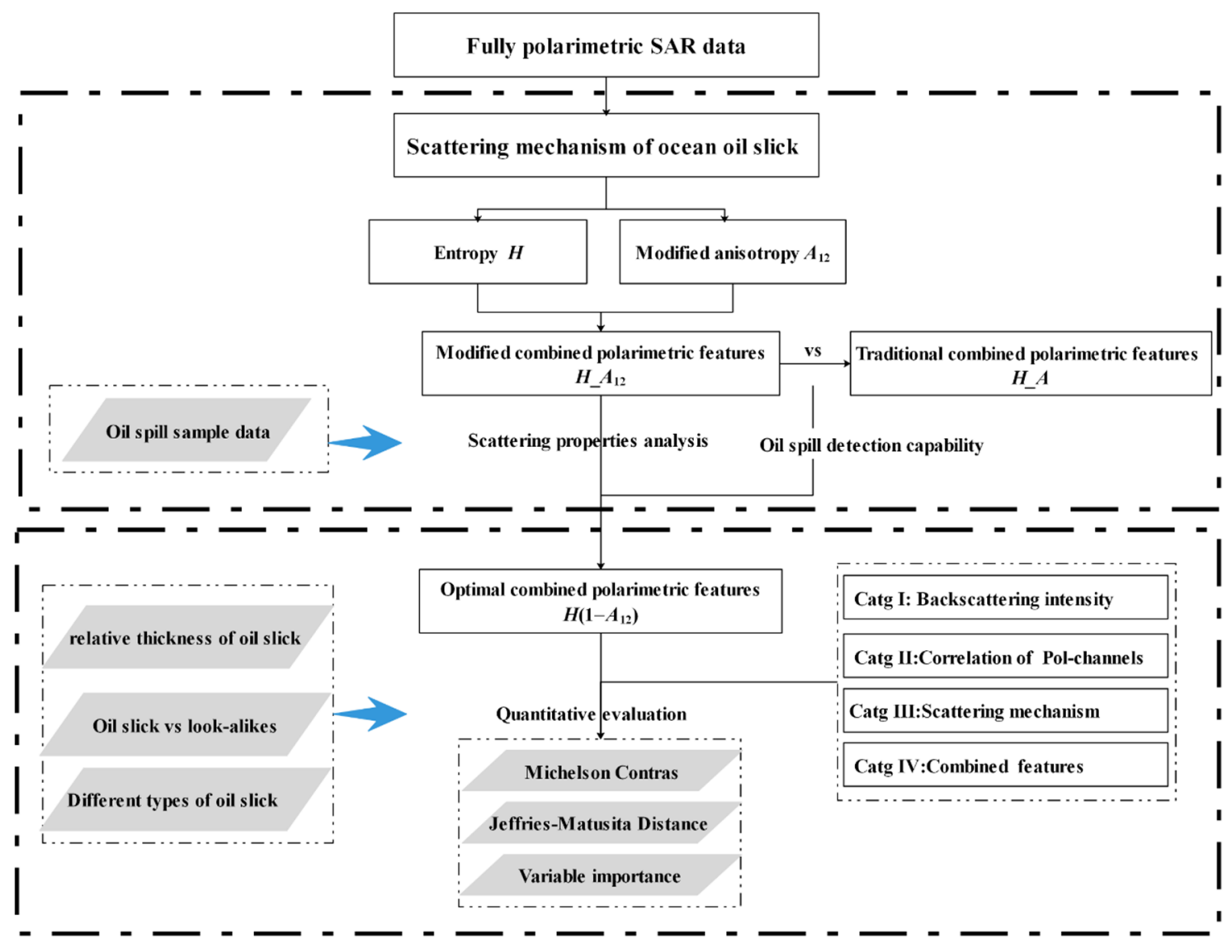

Figure 3. Flow charts outlining the proposed method.

\subsection{Fully Polarimetric SAR Theory}

The full polarimetric SAR system measures four combinations of liner transmit and receive polarization channel $[10,13]$. The potential and advantages of full polarimetric SAR in interpreting and detecting marine oil slick have been demonstrated under different oil spill scenarios by many prior studies $[6,9,11-13]$. The full scattering matrix $S$ and its 
corresponding Pauli scattering vector $\mathrm{k}$ under the fully polarimetric SAR system are given as [10]:

$$
S=\left[\begin{array}{ll}
S_{H H} & S_{H V} \\
S_{V H} & S_{V V}
\end{array}\right] \Rightarrow \boldsymbol{k}=\operatorname{Vector}(S)=\frac{1}{\sqrt{2}}\left[S_{H H}+S_{V V}, S_{H H}-S_{V V}, 2 S_{H V}\right]^{\mathrm{T}}
$$

where $T$ denotes transpose. The scattering matrix elements $S_{x y}$, with $x, y \in\{H, V\}$, where $x$ and $y$ represent transmit and receive, and $H$ and $V$ represent horizontal and vertical polarizations, respectively. Assuming reciprocity, the relation $S_{H V}=S_{V H}$ is satisfied, and the Pauli-basis scattering vector $k$ (the dimension is 3 ) contains the same information as the scattering matrix $S$ [10].

The $3 \times 3$ complex coherence matrix $T_{3}$ can be constructed based on the threedimensional Pauli-basis scattering vector $k$ and its conjugate transpose $k^{* T}$, which can extract a series of effective polarimetric features and describe properties of the scattering target. The form of matrix $T_{3}$ are given by [10]:

$$
=\frac{1}{2}\left[\begin{array}{ccc}
T_{3}=\left\langle k \cdot k^{* T}\right\rangle & \\
\left\langle\left|S_{H H}+S_{V V}\right|^{2}\right\rangle & \left\langle\left(S_{H H}+S_{V V}\right)\left(S_{H H}-S_{V V}\right)^{*}\right\rangle & 2\left\langle\left(S_{H H}+S_{V V}\right) S_{H V}^{*}\right\rangle \\
\left\langle\left(S_{H H}-S_{V V}\right)\left(S_{H H}+S_{V V}\right)^{*}\right\rangle & \left\langle\left|S_{H H}-S_{V V}\right|^{2}\right\rangle & 2\left\langle\left(S_{H H}-S_{V V}\right) S_{H V}^{*}\right\rangle \\
2\left\langle S_{H V}\left(S_{H H}+S_{V V}\right)^{*}\right\rangle & 2\left\langle S_{H V}\left(S_{H H}-S_{V V}\right)^{*}\right\rangle & 4\left\langle\left|S_{H V}\right|^{2}\right\rangle
\end{array}\right]
$$

Here, $<\cdot>$ denotes the ensemble averaging. Polarimetric features can be obtained using $T_{3}$ matrices. Eigenvalues or eigenvectors can be computed from the Hermite averaged coherence matrix $T_{3}$, which can be further expanded into sets of polarimetric features, described, interpreted, and analyzed to obtain the polarization information of targets. The decomposition process for Hermite averaged coherence matrix $T_{3}$ is given as follows [10]:

$$
\left\langle T_{3}\right\rangle=U_{3}[\Sigma] U_{3}^{-1}=\sum_{i=1}^{i=3} \lambda_{i} T_{i}=\sum_{i=1}^{i=3} \lambda_{i} u_{i} u_{i}^{T *}
$$

Here, $\sum$ represents the three-dimensional diagonal matrix, and $u_{i}$ and $\lambda_{i}$ are the orthogonal unit eigenvectors and eigenvalues of the coherent matrix, respectively.

In this paper, the parametric analysis of RADARSAT-2 data is primarily grounded in the eigenvalue- and eigenvector-based Cloude polarization decomposition algorithm, including $H / A$ calculated by [25], anisotropy $A_{12}$ revised by [6], and traditional $H \_A$ combination introduced by $[18,24]$. Among them, entropy, $H$, represents the degree of randomness of the scattering mechanisms that dominate the target, which is one component of the classic $H / A$ / $\alpha$ decomposition and is defined by a logarithmic form of eigenvalues $\lambda_{i}$ obtained from $T_{3}\left(\lambda_{1}>\lambda_{2}>\lambda_{3}\right)$, and is given as follows [10,25]:

$$
\begin{aligned}
& H=-\sum_{i=1}^{3} P_{i} \log _{3} P_{i} \\
& P_{i}=\frac{\lambda_{i}}{\lambda_{1}+\lambda_{2}+\lambda_{3}}
\end{aligned}
$$

The conventional $A$ can be considered as the effective parameter for measuring smallscale roughness on the sea surface, which is calculated using the two largest eigenvalues. The form of $A$ is given by [10]:

$$
A=\frac{\lambda_{2}-\lambda_{3}}{\lambda_{2}+\lambda_{3}}
$$

The modified anisotropy $A_{12}$ is calculated using the two largest eigenvalues of the coherence matrix, differing from the traditional anisotropic $A$ defined by the two smallest 
eigenvalues [6]. Studies using a range of different oil spill scenarios have demonstrated the effectiveness and robustness of $A_{12}$, and is given as follows:

$$
A_{12}=\frac{\lambda_{1}-\lambda_{2}}{\lambda_{1}+\lambda_{2}}
$$

\subsection{Construction Frame of Combined Polarimetric Features}

To enable oil spill detection, polarimetric features quantify and visualize differences between targets based on the mechanisms of scattering and scattering characteristics of the oil slick. High-quality polarimetric features can effectively highlight the oil slick signal and suppress false alarm in different oil spill scenarios. In recent years, extensive application and expansion of polarization data has significantly improved the employment of traditional parameters. Combined polarimetric features can be considered the characteristics of different constituent parameters and enhance separability between different targets through mathematical transformations; this has been effectively applied in oil spill detection $[26,27]$. Schuler constructed the $H \_A$ combination polarimetric feature based on the $H$ and $A$ parameters, conducted oil spill detection analysis, and explored the features that could lead to an increase in the probability of oil spill detection according to several mathematical combinations of parameters, including $H A, H(1-A), A(1-H)$, and $(1-H)(1-A)$ [18]. Cai compared and analyzed the scattering properties of oil spills based on combination feature spectra composed of entropy $H$ and anisotropic $A$ [26]. Zou further used the combination of $H$ and $A$ to detect oil spills, verifying the effectiveness of this combination of polarimetric features [27].

$$
H \_A=\left\{\begin{array}{c}
H A \\
H(1-A) \\
A(1-H) \\
(1-H)(1-A)
\end{array}\right.
$$

Furthermore, other studies have introduced improved and modified feature parameters on the polarization feature space. This study proposes an improved $H_{-} A_{12}$ combination feature based on the traditional $H \_A$ combination, which comprehensively considers ability to distinguish oil slicks using entropy $H$ and improved anisotropy $A_{12}$, and the expansion ability of the combination parameter [2]. A new set of combination polarimetric features $H_{-} A_{12}$ is generated, which is defined as follows:

$$
H_{-} A_{12}=\left\{\begin{array}{c}
H A_{12} \\
H\left(1-A_{12}\right) \\
A_{12}(1-H) \\
(1-H)\left(1-A_{12}\right)
\end{array}\right.
$$

As per the theoretical polarimetric features of oil spills and seawater in $H$ and $A_{12}$, $H\left(1-A_{12}\right)$ represents the random scattering process of a target, highlighting target signals with high entropy and low improved anisotropy values, such as the oil slick; however, at the same time, it effectively suppresses false alarm clutter signals with low randomness, such as background seawater and look-alikes with weak damping effects. Therefore, the theoretical mechanism is aligned with the purpose of the application and the requirements of oil spill detection. Considering case 1 as an example, Figure 4 shows the 3D visualization construction result of the combination feature $H\left(1-A_{12}\right)$. Based on different target, samples were randomly selected within the study area, and the resulting scatter distribution of the $2 \mathrm{D}$ space between the combination setting and the corresponding single component setting is shown in Figure 5. This shows that the same target signal, $H\left(1-A_{12}\right)$, has a larger span of quantitative values than either $H$ or $A_{12}$, prompting a larger contrast between targets because of the mathematical function taking the form of a product between two sub-parameters. 


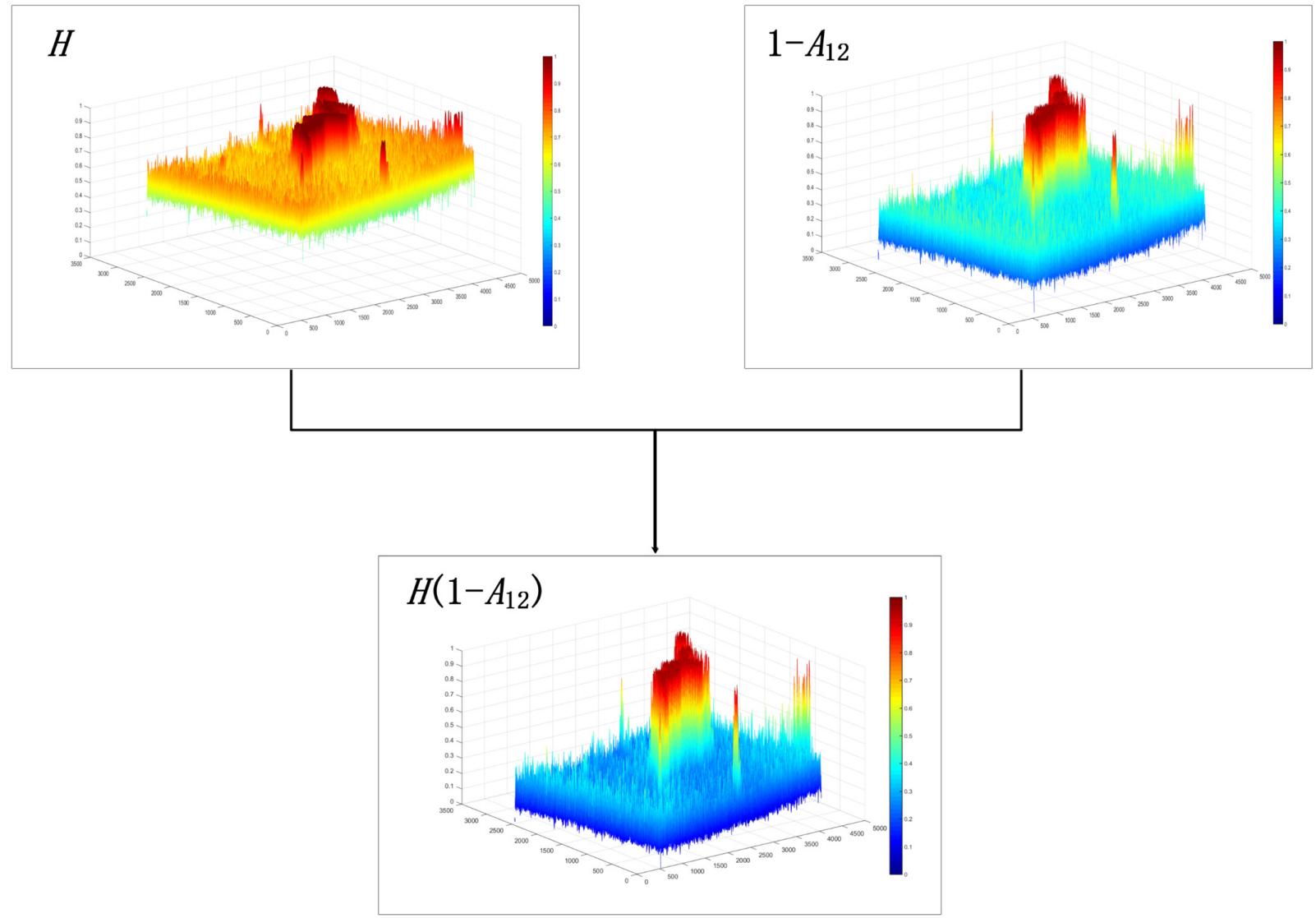

Figure 4. Schematic showing the combined polarimetric parameter $H\left(1-A_{12}\right)$ of natural oil seeps in case 1.

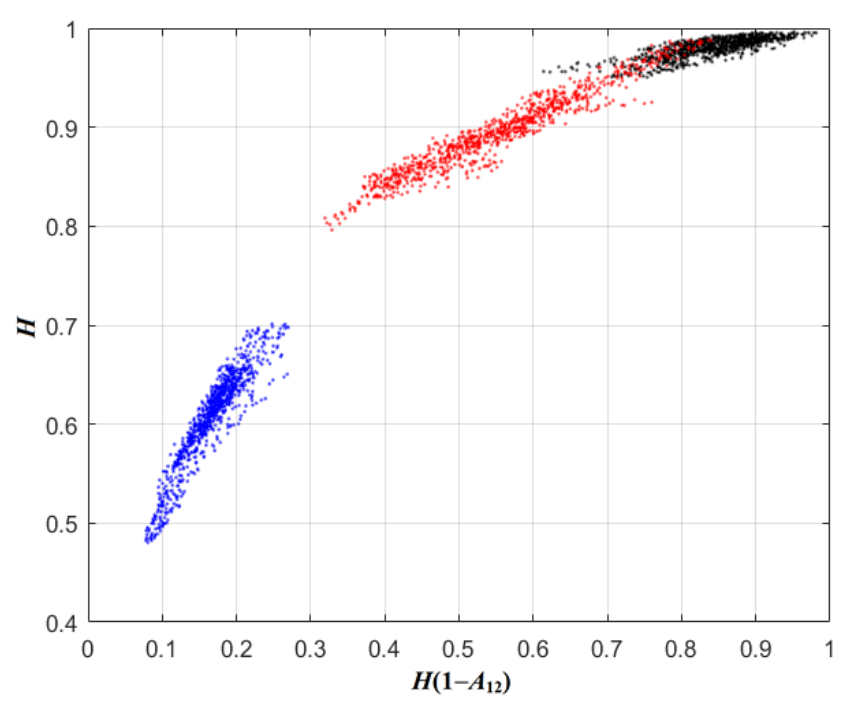

(a)

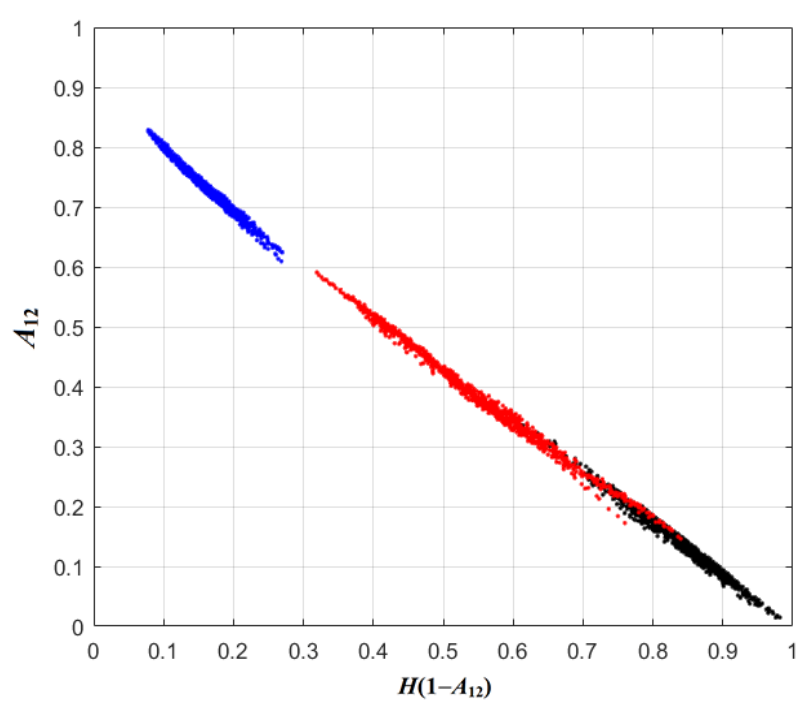

(b)

Figure 5. Scatter plots of combination parameter versus corresponding single-component parameters: (a) $H\left(1-A_{12}\right)$ vs. $H$; (b) $H\left(1-A_{12}\right)$ vs. $A_{12}$. 
It is necessary to confirm the advantages of the proposed combination parameter $H\left(1-A_{12}\right)$ relative to the traditional $H_{-} A$ combination. Considering the relative thickness information of the oil slick as the research objective, qualitative and quantitative analysis was used to compare the ability of the two sets of parameters to distinguish a thick oil slick, a thin oil slick, and seawater. Qualitative analysis was conducted based on visualization results and the histogram distribution of the two sets of parameters. Quantitative analysis was based on the proportion of overlapping areas between the target areas under each polarimetric feature. Figure 6 shows the visualization results of the $H \_A$ combination and the $H \_A_{12}$ combination parameters. With the exception of $(1-H)(1-A)$, the results using combination $H \_A_{12}$ are overall improved compared to those of the $H \_A$ combination, which can be used to suggest an alternate strategy to the latter in oil spill detection applications. Among these, the $H\left(1-A_{12}\right)$ parameter results exhibit the largest contrast between the oil slick and the background seawater, indicating that these parameters result in a better suppression effect on seawater clutter on the basis of increasing the signal intensity of the oil slick target. Moreover, 4000 sample points from various targets were randomly selected using the "Create Random Points" tool with ArcGIS 10.2. Figure 7 shows the histograms and distribution results of the three types of samples in the 1D feature space of the two-set combination. These parameters have not been normalized to maintain the characteristics of the original information and data.

Figure 8 shows the proportion of overlapping areas of target samples based on two sets of combination feature spaces, indicating the cross-mixing phenomena of target samples under their corresponding feature space. At higher overlap ratios, the ability to distinguish between the target samples is weaker and vice versa. Data from the three target samples selected in this section are distributed on the same coordinate scale with the same total area of 4000 units. According to the histogram shown in Figure 8, the overlap area ratios of targets under the $H_{-} A_{12}$ combination parameters are lower than those under the corresponding $H \_A$ combination parameters, with the exception of the $(1-H)(1-A)$ parameters, indicating that the former is superior from the viewpoint of oil spill detection. The two parameters with the lowest overlap area ratios between targets are $H\left(1-A_{12}\right)$ and $(1-H)(1-A)$.

Our results demonstrate that all the parameters have a certain separation between oil and seawater, with the exception of $H A$, which exhibits a high overlap between the oil slick and seawater. Comprehensive comparisons between the statistical distribution of the two datasets shows that the oil-water contrast when using $H_{-} A_{12}$ combination is greater than that when using the $H \_A$ combination, with the exception of $(1-H)(1-A)$; moreover, $H\left(1-A_{12}\right)$ resulted in the largest oil-water separation among the eight combination parameters tested. 

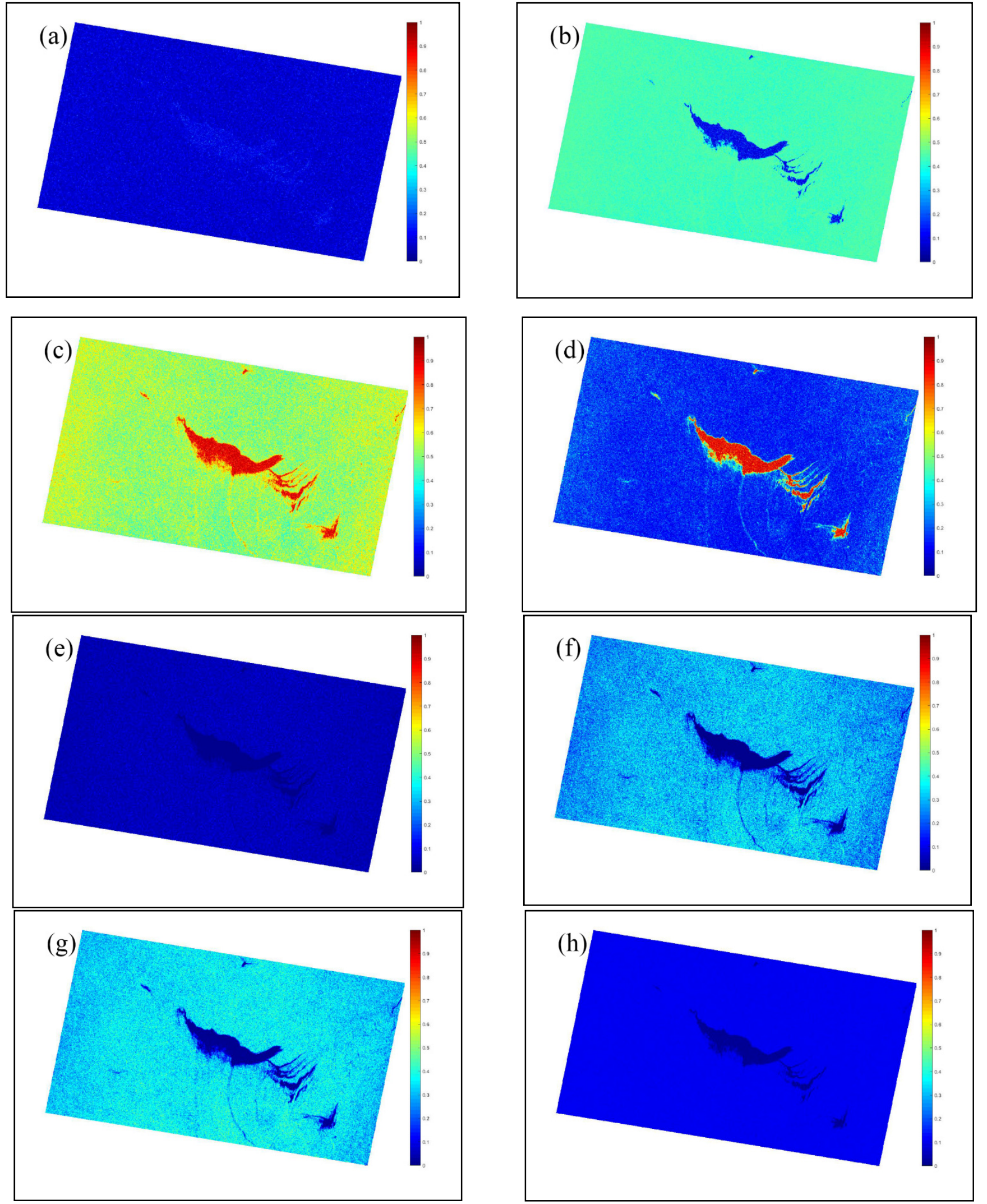

Figure 6. Visual images showing the combination parameters $H \_A$ and $H \_A_{12}$. (a) $H^{*} A$. (b) $H^{*} A_{12}$. (c) $H^{*}(1-A)$. (d) $H^{*}(1-$ $\left.A_{12}\right)$. (e) $A^{*}(1-H)$. (f) $A_{12}{ }^{*}(1-H)$. (g) $(1-H)^{*}(1-A)$. (h) $(1-H)^{*}\left(1-A_{12}\right)$. 

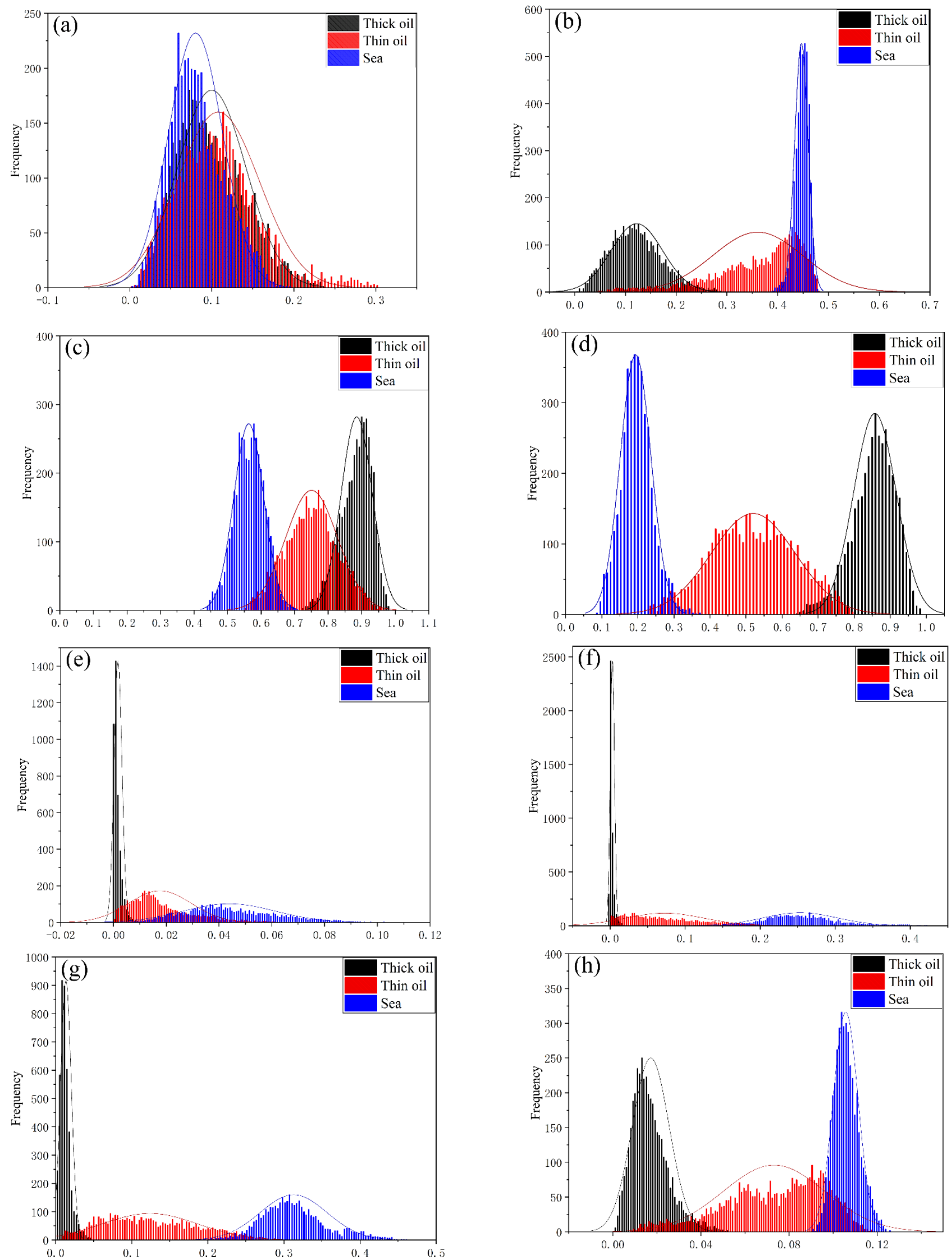

Figure 7. Histograms and distributions of target samples under different combinations of parameters. (a) $H^{*} A$. (b) $H^{*} A_{12}$. (c) $H^{*}(1-A)$. (d) $H^{*}\left(1-A_{12}\right)$. (e) $A^{*}(1-H)$. (f) $A_{12}{ }^{*}(1-H)$. (g) $(1-H)^{*}(1-A)$. (h) $(1-H)^{*}\left(1-A_{12}\right)$. 


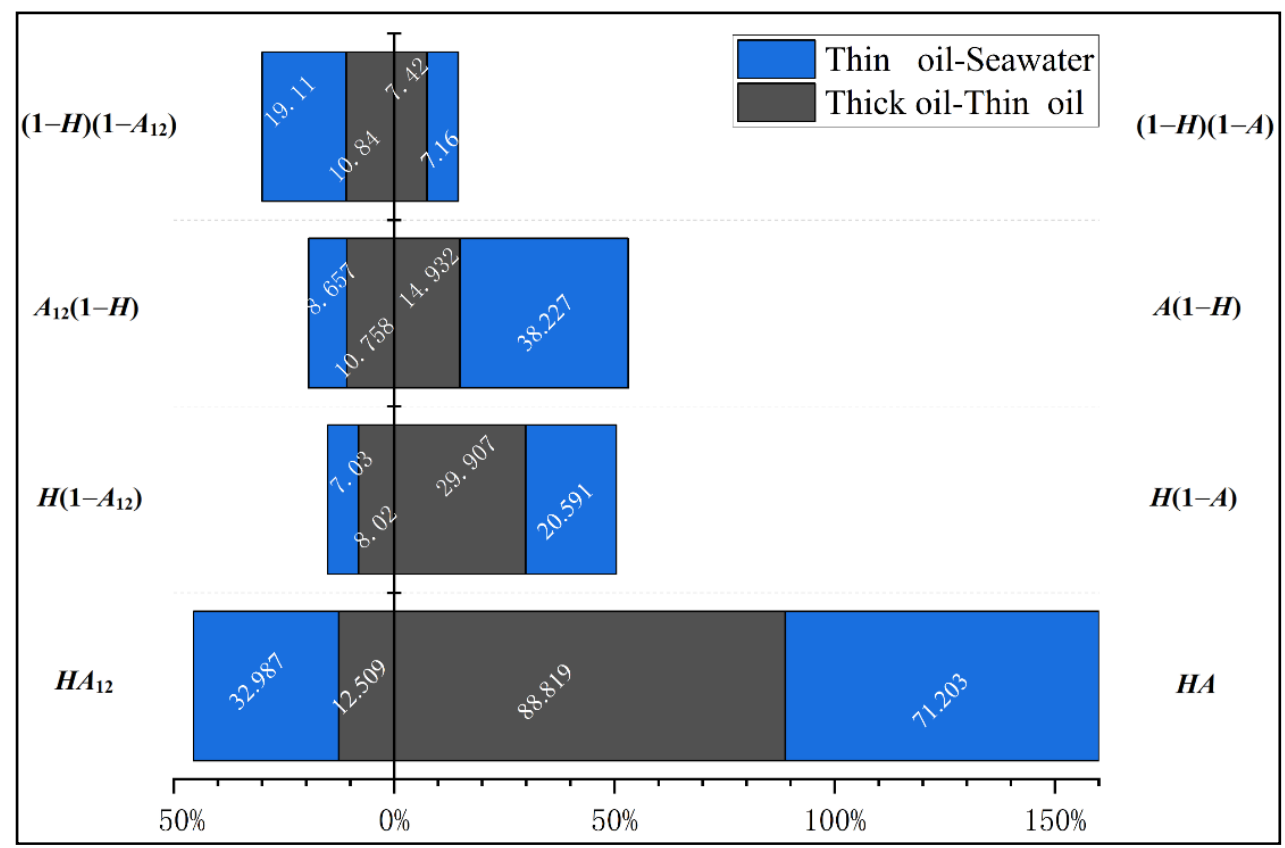

Figure 8. The proportion of overlapping areas between various sample distributions under one-dimensional feature parameters.

\subsection{Oil Spill Detection Ability Comparison of $\mathrm{H}\left(1-A_{12}\right)$}

To evaluate and confirm the advantage and robustness of $H\left(1-A_{12}\right)$ for oil spill detection, we quantitatively compared the results of $H\left(1-A_{12}\right)$ against those from other types polarimetric features based on three groups of oil spill scenarios using polarization SAR data: relative thickness information of oil slicks, oil slicks versus look-alikes, and different types of oil slicks. As mentioned above, polarimetric feature parameters mainly include four types: backscattered energy, scattering mechanism, correlation between channels, and combination feature. The four types of general polarimetric features investigated in this study can be computed and expanded based on the Equations (1)-(7) in Section 3.1, which are respectively introduced by references $[6,8,17,28-31]$. Table 3 lists the four types of polarimetric feature commonly used in oil spill detection compared to $H\left(1-A_{12}\right)$ in this study, their definitions formula, corresponding references, abbreviations, and expected behavior over sea with and without oil slicks.

Table 3. Details of the four categories of general polarimetric features investigated in this study, including their reference, definitions and expected behavior over oil slicks and the sea.

\begin{tabular}{|c|c|c|c|c|}
\hline Categories & Abbreviation/Reference & Definition & Oil Slick & Sea \\
\hline \multirow{2}{*}{ Backscattered energy } & $\tau[15]$ & $\tau=\left\langle\left|S_{H H}+S_{V V}\right|\right\rangle^{2} / \sum^{i=3} \lambda_{i}$ & Low & High \\
\hline & $P R[6,8,28]$ & $P R=\left\langle\left|S_{H H}\right|\right\rangle^{2} /\left\langle\left|S_{V V}\right|\right\rangle^{2}$ & High & Low \\
\hline \multirow{2}{*}{ Scattering mechanism } & $A_{12}[6]$ & $A_{12}=\left(\lambda_{1}-\lambda_{2}\right) /\left(\lambda_{1}+\lambda_{2}\right)$ & Low & High \\
\hline & $H[8,29]$ & $H=\sum_{i=1}^{3}-P_{\mathrm{i}} \log _{3} P_{i}$ & High & Low \\
\hline \multirow{2}{*}{$\begin{array}{l}\text { Correlation between } \\
\text { channels }\end{array}$} & $p_{c o}[6,30,31]$ & $\rho_{-}$co $=\left|\left\langle\left|S_{H H} S_{V V}^{*}\right|\right\rangle\right| /\left|\sqrt{\left\langle\left|S_{H H}\right|\right\rangle^{2}\left\langle\left|S_{V V}\right|\right\rangle^{2}}\right|$ & Low & High \\
\hline & $r_{c o}[6]$ & $r_{c o}=\left|\operatorname{Re}\left(\left\langle S_{H H} S_{V V}^{*}\right\rangle\right)\right|$ & Low & High \\
\hline \multirow{2}{*}{ Combination feature } & $F[17]$ & $F_{\text {wang }}=\left[(1-H)+(1-\alpha)+A_{12}+\rho_{-} c o\right] / 4$ & Low & High \\
\hline & $H\left(1-A_{12}\right)$ & $H\left(1-A_{12}\right)$ & High & Low \\
\hline
\end{tabular}


Three quantitative assessment methods were selected based on their widespread used in oil spill detection, i.e., the Michelson comparison measure, Jeffreys-Matusita distance, and input variable importance assessment based on the random forest classification module $[6,13,32-35]$.

\subsubsection{Michelson Contrast}

The basic perceptual attributes of an image are based on obvious and perceived contrast between target samples. In previous research, contrast sensitivity was used as the main content of visual perception research. Various contrast assessment indexes have been developed and improved to facilitate improved digital image processing, amongst which the Michelson contrast is one of the general criteria for evaluating target separability. It has thus been used to quantitatively define and evaluate contrasts between oil slicks and seawater under various polarimetric feature spaces $[6,34]$ :

$$
M C=\frac{I_{\max }-I_{\min }}{I_{\max }+I_{\min }}
$$

where $I_{\max }$ and $I_{\min }$ represent the maximum and minimum mean polarimetric feature values between the two target samples being tested, respectively, and the value range of $\mathrm{MC}$ is $[0,1]$.

\subsubsection{Jeffries-Matusita Distance}

The Jeffries-Matusita (J-M) distance is an extensively used statistical separability index for selecting optimal features in the field of oil slick detection based on Pol-SAR imaging, which is itself a simple and easily implemented criterion [13,15,33,36,37]. The $\mathrm{J}-\mathrm{M}$ distance has been demonstrated to be effective, easily implemented, and with an appropriate level of universality to conduct pairwise measurements of the separability between oil slicks and seawater in polarimetric feature selection [13,37]. In this study, the $\mathrm{J}-\mathrm{M}$ distance was selected as the measure of separability based on the ability of polarimetric features to distinguish between targets [33]. J-M separability is typically measured using the assumption that the tested target samples have a multivariate normal distribution. In this case, the expression of the J-M distance between two classes $c_{i}$ and $c_{j}$ is defined as follows [33,38]:

$$
J M_{i, j}=2\left(1-e^{-D_{i j}}\right)
$$

where $J M_{i, j}$ represents the J-M distance value for a certain polarimetric feature; $D_{i j}$ represents the Bhattacharyya distance between the class $c_{i}$ and class $c_{j}$, which is introduced by [33] and takes the form:

$$
D_{i j}=\frac{1}{8}\left(m_{i}-m_{j}\right)^{T}\left[\frac{\Sigma_{i}+\Sigma_{j}}{2}\right]^{-1}\left(m_{i}-m_{j}\right)+\frac{1}{2} \ln \left(\frac{\left|\frac{\Sigma_{i}+\Sigma_{j}}{2}\right|}{\sqrt{\left|\Sigma_{i}\right|\left|\Sigma_{j}\right|}}\right)
$$

where $m_{i}$ and $\sum_{i}$ represent the mean vector and covariance matrices of $c_{i}$ samples, which are members of a set of $C$ classes in a certain feature space, and $m_{j}$ and $\sum_{j}$ represent the mean vector and covariance matrices of $c_{j}$ samples, respectively [33]. The first term in the $D_{i j}$ is similar to the square of the normalized distance between the mean of the class. Because of the contribution of the intrinsic properties of the exponential factor, the J-M distance exhibits saturation behavior as the separability of targets increases, and thus provides an exponentially decreasing weight to increase the separability. The J-M distance most closely represents the probability of a correct classification, and can therefore provide more reliable criteria as a function of the separability between classes $[13,33,37,38]$. The value of the J-M distance ranges from 0 to 2 . High $\mathrm{J}-\mathrm{M}$ distances denote a greater degree of separability between different ground targets and vice versa. When the J-Mdistance is greater than 1.9, 
the two ground targets have strong separability; values of 1-1.9 indicate good separability, whereas those ranging from 0 to 1 indicate weak separability $[4,37]$.

\subsubsection{Variable Importance}

Random Forest is an extensively used supervised learning method that integrates multiple classification and regression trees, and has been extensively used in polarimetric SAR oil spill detection. Random forest includes two main parts: tree growth and vote. Moreover, it follows two randomness rules: the random selection of the training sample subset and the random selection of the feature subset [39,40]. Furthermore, the RF module includes two methods for quantitative assessments of the importance of input feature variables: MDI (mean decrease impurity) and MDA (mean decrease accuracy) [32]. In this study, MDA indexes were used to evaluate and rank the importance of input variables. EnMAP-Box is a toolbox developed for processing and analyzing data acquired by the German spaceborne imaging spectrometer EnMAP (Environmental Mapping and Analysis Program), which considers the standards of general-purpose imaging data analysis software and the extending functionality of machine learning [41,42]. In this study, EnMAP-Box was utilized with two objectives to guarantee full comparison between the differences of polarimetric features:

(i) Obtain oil spill detection results under different oil spill scenarios based on the RF module;

(ii) Evaluate the contribution of different types of polarimetric features to the corresponding classification results.

\section{Experiment Result and Analysis}

\subsection{Michelson Contrast Results}

Figure 9 shows the MC measure evaluation results of the studied four types polarimetric features under different oil spill scenarios, which shows the ranking results between the thickness of an oil slick (a), the oil slick and its look-alike (b), and different types of oil slicks (c) (d). Overall, $H\left(1-A_{12}\right)$ results in good separation between different oil spill scenarios, and is better than other polarimetric features in most cases because it indicates relatively large differences among targets. The $A_{12}$ and $H$ parameters have good distinguishing ability, but their performance with respect to scattering energy features is relatively poor. In research regarding the identification of the relative thickness of an oil slick, $H\left(1-A_{12}\right)$ exhibits the best overall performance; however, the results for distinguishing between the thick and thin oil slick are slightly lesser than those of $A_{12}, F$, and $p_{-c o}$. Furthermore, the $\mathrm{MC}$ results are optimal between the oil slick and its look-alike, between different types of oil slicks and seawater, and between different types of oil slicks.

\subsection{J-M Distance Results}

Figure 10 shows the J-M distances for the studied regions. J-Mmeasurement results of the four types of polarimetric features between the relative thickness information of the oil slick are generally similar to MC results. $H\left(1-A_{12}\right)$ achieves optimal or close to optimal results. $H$ performs well in $\mathrm{MC}$ measures among numerous types of target; however, the overall $P R$ measure presents relatively low MC results. Between the oil slick and lookalike, the J-Mdistance result of $H\left(1-A_{12}\right)$ presents the optimal result; however, it is less effective in distinguishing between seawater and look-alikes, indicating that $H\left(1-A_{12}\right)$ can highlight oil slick signals while suppressing false alarm signals, such as sea clutter and look-alikes. $H$ and $H\left(1-A_{12}\right)$ both show better results between different types of oil slick, than between other features. Of the tested methods, $H\left(1-A_{12}\right)$ showed good overall robustness, with optimal or close to optimal results among all targets. Nonetheless, its J-M results between plant oil and seawater were slightly lower, indicating that $H\left(1-A_{12}\right)$ most effectively identifies mineral oil slicks. 


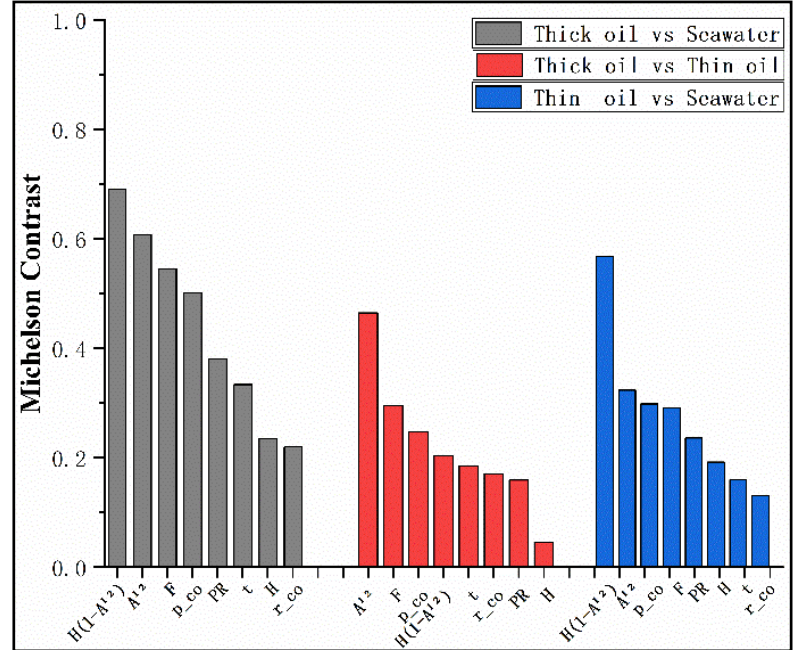

(a)

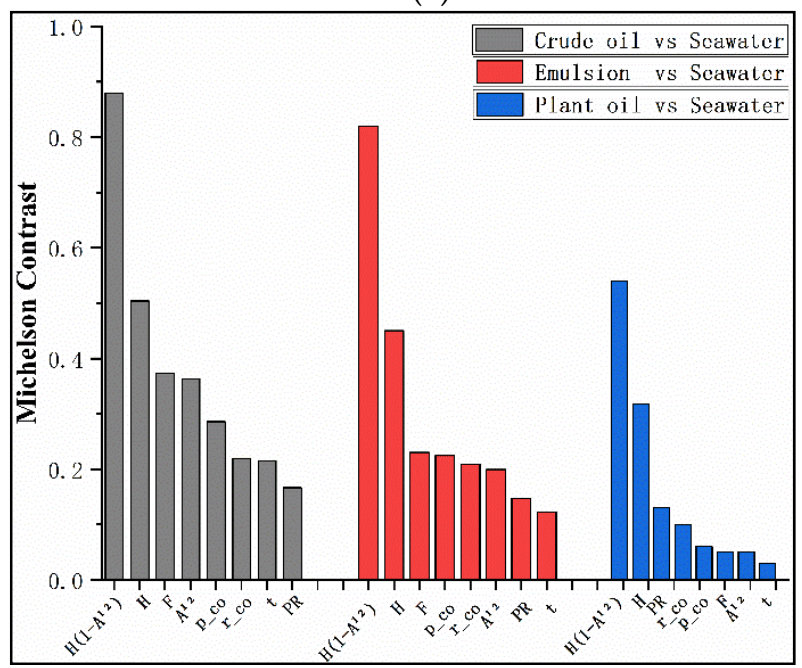

(c)

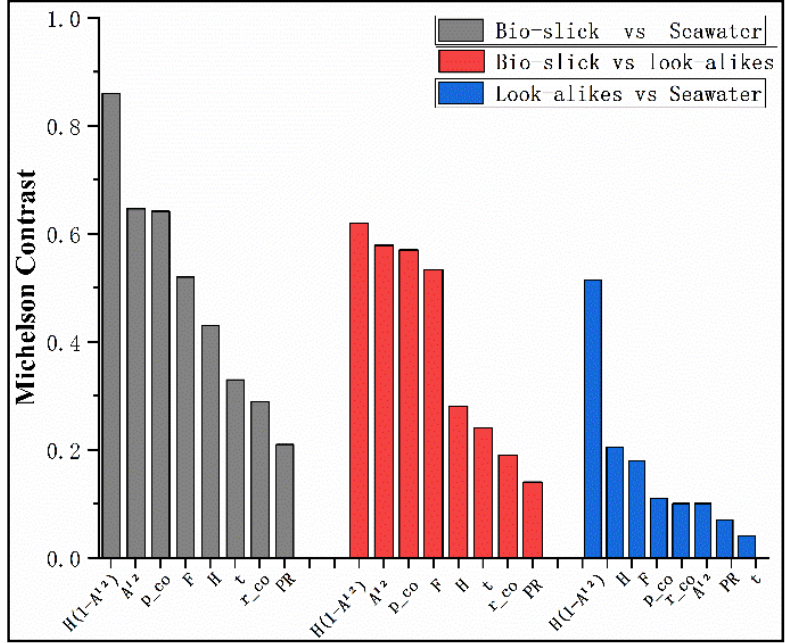

(b)

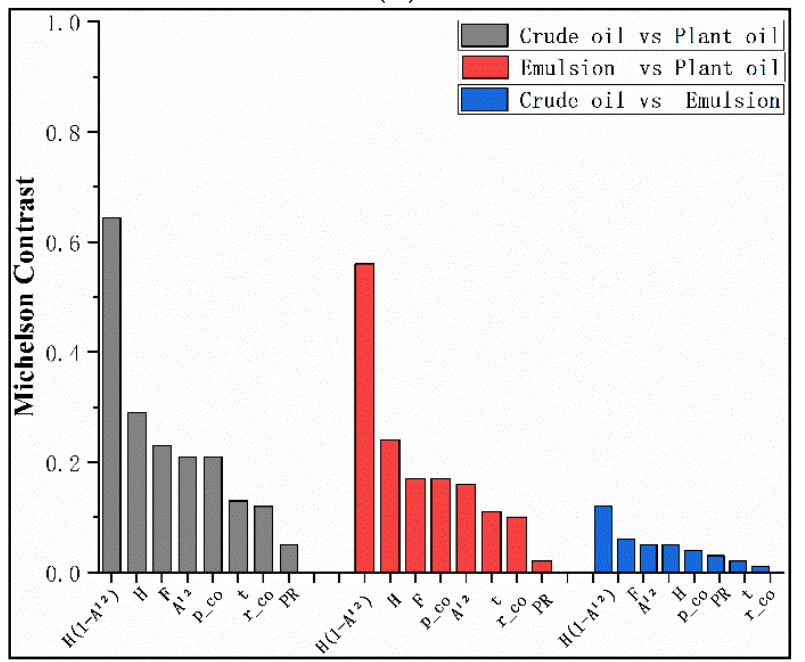

(d)

Figure 9. MC measurement results with respect to different types of polarimetric feature. (a) Relative thickness of oil slick. (b) Oil slick vs look-alikes. (c) Different types of oil slick versus seawater. (d) Different types of oil slick.

\subsection{Variable Importance Ordering Results}

The oil spill detection results and the variable importance ordering under relative thicknesses of oil slicks are shown in Figure 11 and Table 4. Thick oil slicks, thin oil slicks, and seawater are successfully classified with good producer and user accuracy results. However, the results feature certain misclassifications, especially the thin oil slick, which resulted in a slightly lower Kappa coefficient. Based on the effective classification results, $H\left(1-A_{12}\right)$ has the highest contribution, followed by $H$ and $A_{12}$, demonstrating the advantages of $H\left(1-A_{12}\right)$ in extracting information regard the relative thickness of an oil lick. 


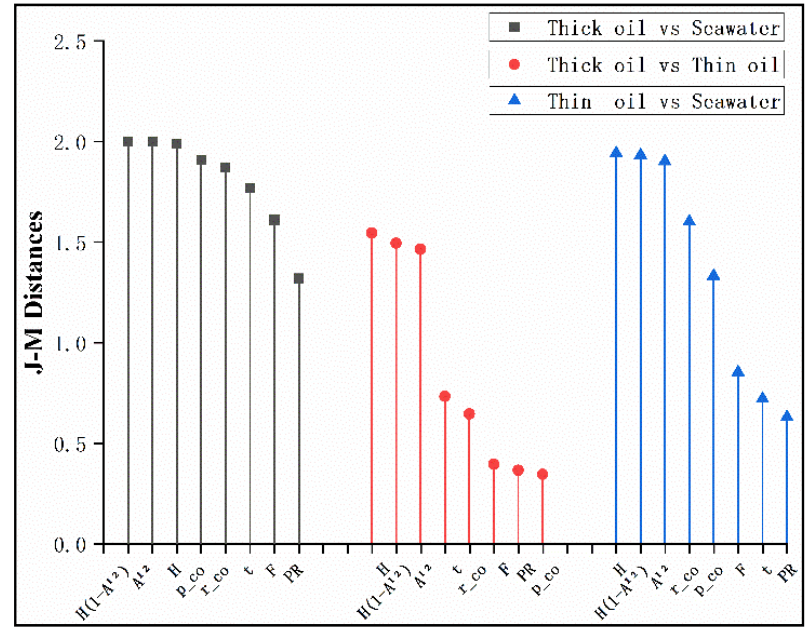

(a)

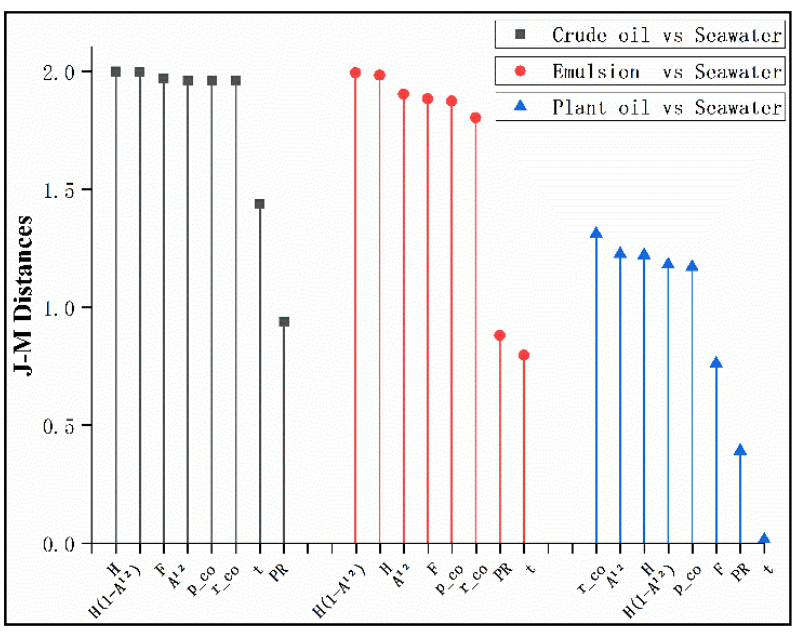

(c)

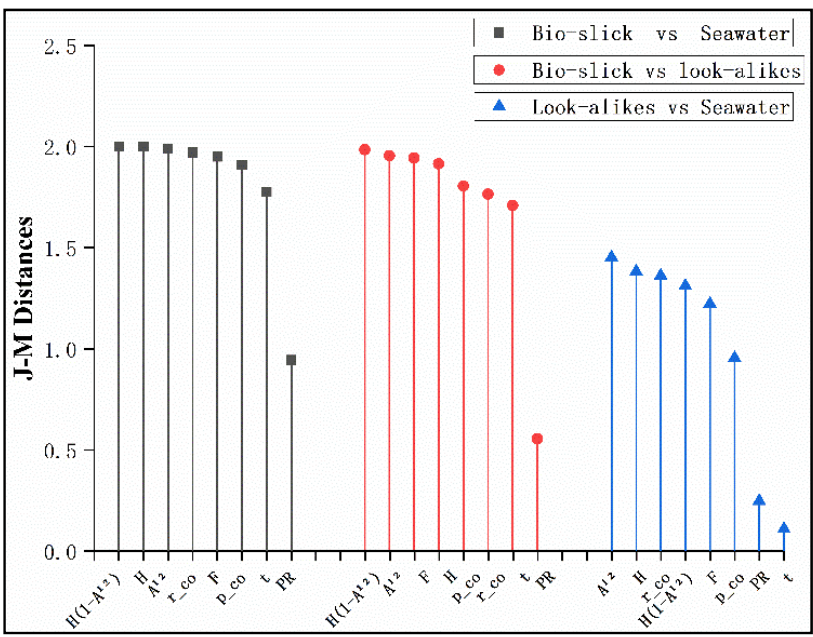

(b)

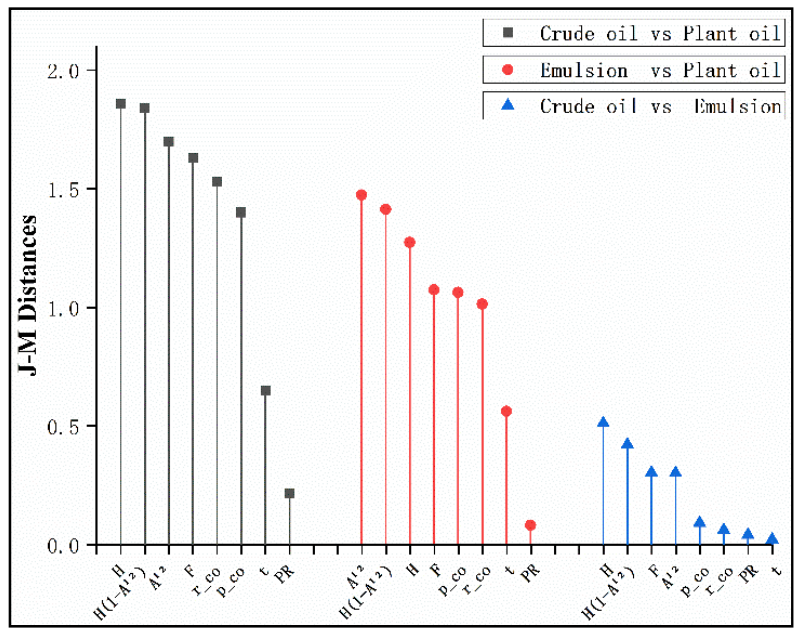

(d)

Figure 10. J-Mdistance measurement results for different types of polarimetric feature. (a) Relative thickness of oil slick. (b) Oil slick vs. look-alikes. (c) Different types of oil slick versus seawater. (d) Different types of oil slick.

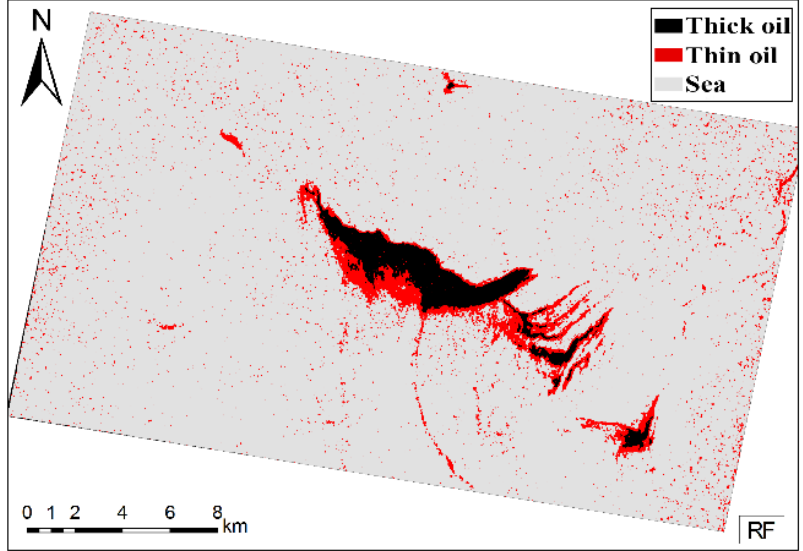

(a)

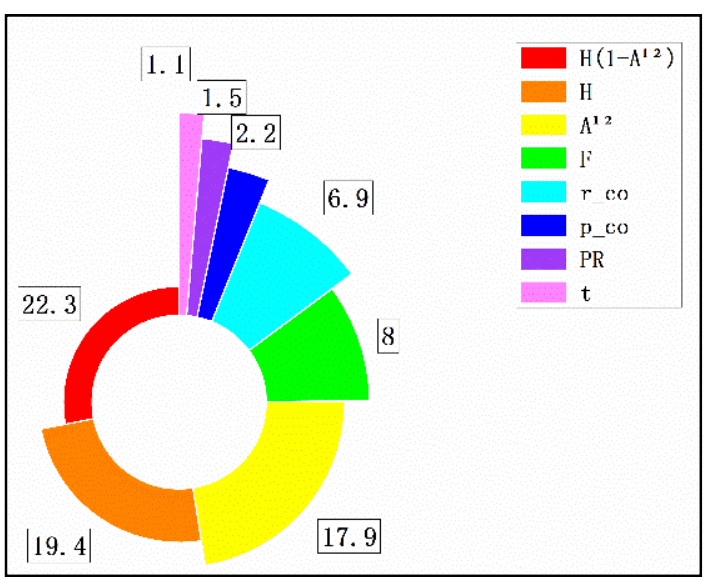

(b)

Figure 11. Results of the Random Forest classifier and importance ordering of polarimetric features in study area 1: (a) oil spill detection results; (b) variable importance ordering. 
Table 4. Classification accuracies of the Random Forest classifier in study area 1.

\begin{tabular}{cccc}
\hline Class $\backslash$ Accuracy & Thick Oil & Thin Oil & Seawater \\
\hline Producer Accuracy & $97.45 \%$ & $82.77 \%$ & $98.18 \%$ \\
\hline User's Accuracy & $95.53 \%$ & $25.48 \%$ & $99.98 \%$ \\
\hline Average Accuracy & & $83.18 \%$ & \\
\hline Kappa & & 0.76 & \\
\hline
\end{tabular}

Oil spill detection results and the variable importance ordering of the scenario including oil slicks and look-alikes are shown in Figure 12 and Table 5, respectively. Overall, the visualization results and accuracy indicators show that biogenic slicks, look-alikes, and seawater were well classified and extracted such that only the boundary area of the oil slick was sometimes misclassified as a look-alike. Moreover, $H\left(1-A_{12}\right)$ makes a greater contribution than the other four types of polarimetric feature, followed by $H, F$, and $A_{12}$, which indicates good target recognition ability for polarimetric features with distinct types of scattering mechanisms and combination parameters.

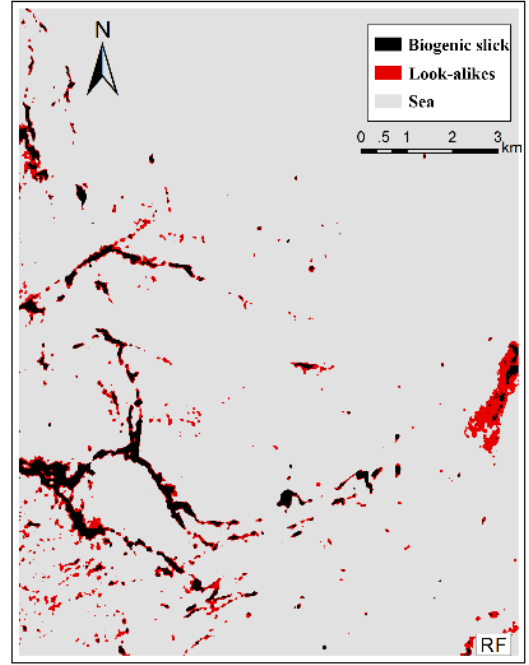

(a)

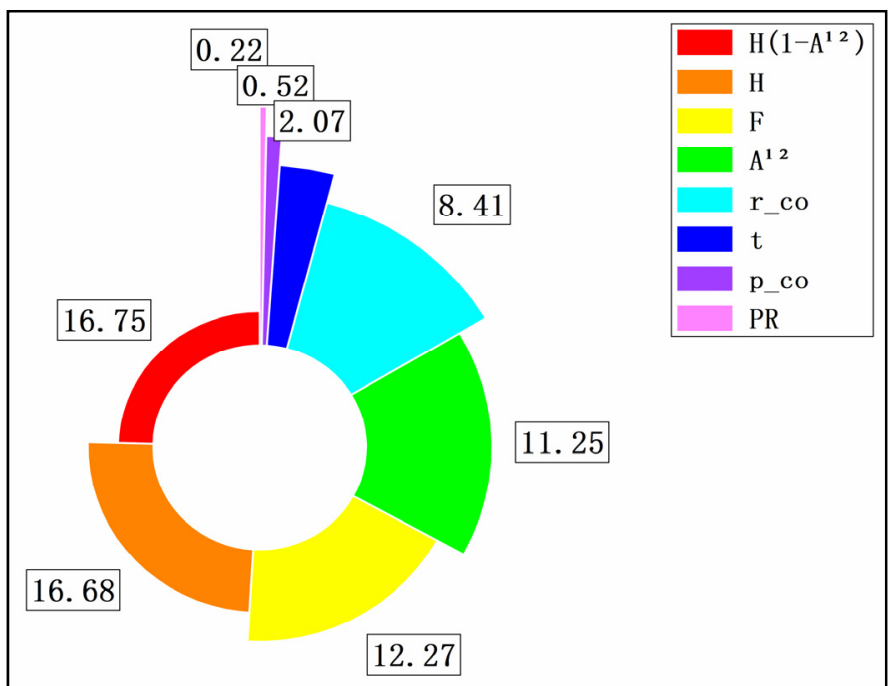

(b)

Figure 12. Results of the Random Forest classifier and importance ordering of polarimetric features in study area 2: (a) oil spill detection results; (b) variable importance ordering.

Table 5. Classification accuracies of the Random Forest classifier in study area 2.

\begin{tabular}{cccc}
\hline Class $\backslash$ Accuracy & Biogenic Slick & Look Alikes & Seawater \\
\hline Producer Accuracy & $83.93 \%$ & $89.92 \%$ & $99.69 \%$ \\
\hline User's Accuracy & $95.29 \%$ & $73.44 \%$ & $99.74 \%$ \\
\hline Average Accuracy & & $90.3 \%$ & \\
\hline Kappa & & 0.9094 & \\
\hline
\end{tabular}

Oil spill detection results and the variable importance ordering of scenarios with different types of oil slicks are shown in Figure 13 and Table 6, respectively. Overall, these visualization results and accuracy indicators demonstrate that mineral oil, plant oil, and seawater were classified and extracted; however, the edge of the crude oil and emulsion and local cavity areas within the thin oil slick were classified as plant oil slicks because their scattering characteristics are similar because of weathering. Thicker parts of emulsion were classified as crude oil due to similar phenomena in previous studies $[6,13]$. The most 
effective polarimetric features were $H, H\left(1-A_{12}\right)$ and $A_{12}$. Their importance scores were $29.15,28.4$ and 23.79 , which showed significantly higher performance than subsequent polarimetric features. $H\left(1-A_{12}\right)$ reached a contribution close to the optimal parameter; however, the contributions of scatter power type features were relatively low.

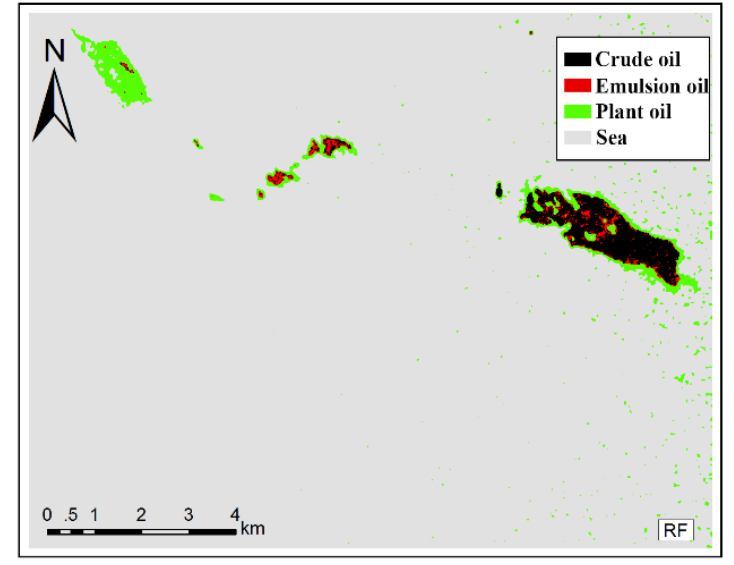

(a)

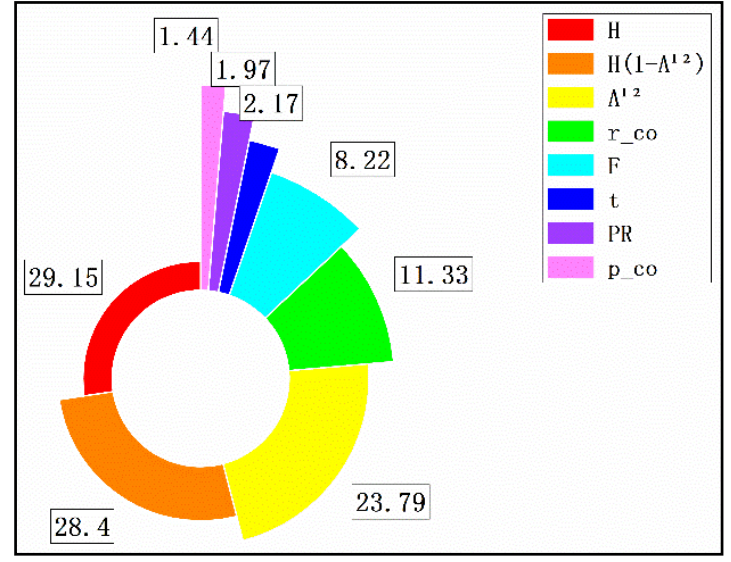

(b)

Figure 13. Results of the Random Forest classifier and importance ordering of polarimetric features in study area 3: (a) oil spill detection results; (b) variable importance ordering.

Table 6. Classification accuracies of the Random Forest classifier in study area 3.

\begin{tabular}{ccccc}
\hline Class $\backslash$ Accuracy & Crude & Emulsion & Plant & Seawater \\
\hline Producer Accuracy & $91.3 \%$ & $63.46 \%$ & $94.81 \%$ & $98.66 \%$ \\
\hline User's Accuracy & $90.77 \%$ & $67.44 \%$ & $78.54 \%$ & $98.04 \%$ \\
\hline Average Accuracy & & $85.37 \%$ & & \\
\hline Kappa & 0.89 & & \\
\hline
\end{tabular}

To summarize, this study comprehensively analyzes the oil spill detection capabilities of four types of polarimetric features considering several different oil spill scenarios, including the relative thickness of oil slicks, oil slicks and look-alikes, and different types of oil slicks. In the MC measure, the combination of polarimetric features $H\left(1-A_{12}\right)$ proposed in this study exhibits optimal overall performance at significantly higher levels than other types of polarimetric feature parameters. This demonstrates that it can effectively improve oil slick signal detection while suppressing sea clutter signals.

\section{Discussion}

\subsection{Analysis of $H_{-} A_{12}$ Combination and $H \_$A Combination}

Effective polarimetric features are primarily manifested through two aspects: highlighting oil slick target signals and effectively suppressing sea clutter and look-alike signals. The proposed method includes two main parts: firstly, the proposed polarimetric feature combination is compared with traditional combinations, and then compared with other types of polarimetric features. Therefore, it effectively uses the scattering mechanism difference between oil slicks and seawater and the expansion capability of polarimetric feature combinations. Firstly, we constructed an improved polarimetric feature combination $H \_A$ based on polarization entropy $H$ and modified anisotropy $A_{12}$, comparing this with traditional combinations $H \_A$ through qualitative and quantitative analysis, and selecting the optimal polarimetric feature combination. Secondly, we compared this polarimetric feature combination with other different types of parameters, confirming its superiority through three different quantitative indicators. Qualitative and quantitative analyses were 
conducted based on visualization results, histogram distribution, and overlapping area proportions between different target samples under suites of polarimetric features. In both sets of parameters, a high degree of overlap between oil slicks and seawater was observed under the $H A$ combination. Almost no overlap was noted between thick oil slicks and seawater; however, certain overlap existed between thin oil and thick oil, as well as between thin oil and seawater. With the exception of $(1-H)(1-A)$, the $H_{-} A_{12}$ combination is superior to $H \_A$ combination in all applications. The minimum overlap area between thick oil slick and thin oil slick was $7.428 \%$ when using $(1-H)(1-A)$, which is only $0.6 \%$ lower than that of $H\left(1-A_{12}\right)$. The minimum overlap area ratio between thin oil slick and seawater is $7.037 \%$ when using $H\left(1-A_{12}\right)$. In general, the $H \_A_{12}$ combination can therefore be used as an alternative to the traditional $H_{-} A$ combination in oil spill detection. Furthermore, $H\left(1-A_{12}\right)$ is the optimal parameter combination when considering both the low overlap proportion and the maximum separation of the sample peak.

\subsection{Analysis of the Advantages of $H\left(1-A_{12}\right)$}

Considering the differences among different oil spill scenarios, it is most effective to perform oil spill detection by utilizing polarimetric features with wide-ranging robustness. Our three quantitative indicator results confirm the superiority and robustness of $H\left(1-A_{12}\right)$ in terms of oil-water relative differences, separation, and classification accuracy. For MC measurement results, $H\left(1-A_{12}\right)$ shows obvious advantages over remaining types of polarimetric feature parameters in diverse oil spill scenarios, especially for distinguishing between different types of oil slicks and seawater because the mathematical combination of $H$ and $A_{12}$ expands the contrast between them. However, the result between thick oil slicks and thin oil slicks is slightly lower than $A_{12}, F$, and $p_{-} c o$. For J-M measurement results, $H\left(1-A_{12}\right)$ can achieve optimal or close to optimal results under different oil spill scenarios; however, the result for distinction between weak damping targets (such as atmospheric front and vegetable oil) and seawater is slightly lower than that of $H$ and $A_{12}$, demonstrating that $H\left(1-A_{12}\right)$ has a good ability to identify mineral oil, but a strong inhibitory effect toward weakly damped targets. For classification applications, $H\left(1-A_{12}\right)$, $H$, and $A_{12}$ showed higher contributions relative to other parameters. In the classification of the relative thickness of oil slicks, $H\left(1-A_{12}\right)$ provides the highest contribution; however, certain portions of the seawater are misclassified as thin oil slicks because of the high randomness of the sea surface in the data, leading to similar scattering characteristics as the thin oil slick. In the oil slick and look-alike scenarios, $H\left(1-A_{12}\right)$ and $H$ showed similarly good results relative to other parameters. Different types of oil slicks were classified, although certain misclassification was noted. The edge and middle regions of thin oil slicks, crude oil, and emulsified oil were misclassified as plant oil because of weathering and diffusion phenomena. Similar results have been observed in other studies using the same data. $H$ presented the highest importance score, followed closely by $H\left(1-A_{12}\right)$. In general, $H\left(1-A_{12}\right)$ effectively improves the acquisition of information about oil spills and suppress weak damping signals. In most oil spill scenarios, $H\left(1-A_{12}\right)$ performs the better or close to the best results than other types of parameters, although $H$ and $A_{12}$ showed good results. Note that although the $H\left(1-A_{12}\right)$ parameter is a combination parameter, its definition and composition both refer to the scattering mechanism types $H$ and $A_{12}$. The overall performance of the scattering mechanism parameters was better, but the scattering energy parameters were lower than in other types of polarimetric features.

\section{Conclusions}

In view of the drawbacks of insufficient exploitation and usage of polarimetric features in oil spill detection, we proposed an improved polarimetric feature combination based on difference in scattering mechanism. The primary contributions of this study are summarized as follows: 
1. Based on the differences inherent to the scattering mechanisms of oil slicks and sea water, an improved polarimetric feature combination suitable for oil spill detection was constructed by combining the polarimetric scattering entropy $H$ and improved anisotropy $A_{12}$, thereby enhancing the contrast between oil slicks and seawater. By comparing the visualization and separation of the improved combination $H_{-} A_{12}$ with the traditional combination $H \_A$, the evaluations and comparisons result demonstrate the superiority of the proposed improved polarimetric feature.

2. Three study sites corresponding to three oil spill scenarios under the same satellite were utilized to evaluate the performance and superiority of the $H\left(1-A_{12}\right)$ combination, including the relative thickness information of oil slicks, oil slicks and look-alikes, and different types of oil slick. The universality and robust performance of $H(1-$ $A_{12}$ ) demonstrates that, when compared with other types polarimetric feature parameters, the proposed $H\left(1-A_{12}\right)$ combination can improve oil-water separation under different oil spill scenarios, thereby improving the acquisition of oil slick information while suppressing sea clutter information.

Future studies will be conducted to confirm the proposed polarimetric feature combining with more remote sensing technology (Hyperspectral, fluorescence or infrared) over a wide range of observation conditions, including different sensor design parameters (image-mode, incidence angle, band, payload platform, NESZ), different properties of oil slicks (oil type, weathering, slick thickness, formation mechanism), different environmental conditions (wind speed, wind direction, climate, different ocean areas), and different lookalike (low wind area, planktons, internal waves, etc.). Moreover, more detailed research on polarimetric feature extraction in the quantitative study and detection of the thickness and spilled amount of marine oil slicks is planned.

Author Contributions: G.L. conceived and performed the experiments; Y.L. and Y.H. supervised and designed the research and contributed to the article's organization; X.W. and L.W. carried out the comparative analysis. G.L. drafted the manuscript, which was revised by all authors. All authors have read and agreed to the published version of the manuscript.

Funding: This article was supported by the project: National Key R\&D Program of China (Grant No.2020YFE0201500), and the Fundamental Research Funds for the Central Universities (grant nos. 3132014302 and 3132019350).

Institutional Review Board Statement: Not applicable.

Informed Consent Statement: Not applicable.

Data Availability Statement: Not applicable.

Acknowledgments: We would like to thank Chen Chen from Dalian Maritime University for comparative analysis in original manuscript. We thank Ridha Touzi and Stefan Nedelcu from the Canada Centre for Remote Sensing for advice regarding data-processing software. We also acknowledge Fengshou Zhang Shiyong Wen and Jianchao Fan of the National Marine Environmental Monitoring Center for processing the data.

Conflicts of Interest: The authors declare no conflict of interest.

\section{References}

1. Fingas, M.; Brown, C. A review of oil spill remote sensing. Sensors 2018, 2, 91.

2. Li, G.; Li, Y.; Liu, B.; Hou, Y.; Fan, J. Analysis of scattering properties of continuous slow-release slicks on the sea surface based on polarimetric synthetic aperture radar. ISPRS Int. J. Geo-Inf. 2018, 7, 237. [CrossRef]

3. Liu, B.; Li, Y.; Liu, C.; Xie, F.; Muller, J.-P. Hyperspectral features of oil-polluted sea ice and the response to the contamination area fraction. Sensors 2018, 2, 234. [CrossRef]

4. Li, G.; Li, Y.; Liu, B.; Wu, P.; Chen, C. Marine oil slick detection based on multi-polarimetric features matching method using polarimetric synthetic aperture radar data. Sensors 2019, 19, 5176. [CrossRef]

5. Migliaccio, M.; Nunziata, F. On the exploitation of polarimetric SAR data to map damping properties of the Deepwater Horizon oil spill. Int. J. Remote Sens. 2014, 35, 3499-3519. [CrossRef] 
6. Skrunes, S.; Brekke, C.; Eltoft, T. Characterization of marine surface slicks by Radarsat-2 multipolarization features. IEEE Trans. Geosci. Remote Sens. 2014, 9, 5302-5319. [CrossRef]

7. Migliaccio, M.; Gambardella, A.; Tranfaglia, M. SAR polarimetry to observe oil spills. IEEE Trans. Geosci. Remote Sens. 2007, 45, 506-511. [CrossRef]

8. Minchew, B.; Jones, C.E.; Holt, B. Polarimetric analysis of backscatter from the Deepwater Horizon oil spill using L-Band synthetic aperture radar. IEEE Trans. Geosci. Remote Sens. 2012, 10, 3812-3830. [CrossRef]

9. Skrunes, S.; Brekke, C.; Doulgeris, A.P. Characterization of low-backscatter ocean features in dual-copolarization sar using log-cumulants. IEEE Geosci. Remote Sens. Lett. 2015, 12, 836-840. [CrossRef]

10. Lee, J.-S.; Pottier, E. Polarimetric Radar Imaging: From Basics to Applications; CRC Press: Boca Raton, FL, USA, 2009.

11. Li, H.; Perrie, W.; He, Y.; Wu, J.; Luo, X. Analysis of the polarimetric SAR scattering properties of oil-covered waters. IEEE J. Sel. Top. Appl. Earth Obs. Remote Sens. 2015, 8, 3751-3759. [CrossRef]

12. Zhang, B.; Perrie, W.; Li, W.; Pichel, W.G. Mapping sea surface oil slicks using RADARSAT-2 quad-polarization SAR image. Geophys. Res. Lett. 2011, 38. [CrossRef]

13. Tong, S.; Liu, X.; Chen, Q.; Zhang, Z.; Xie, G. Multi-feature based ocean oil spill detection for polarimetric sar data using random forest and the self-similarity parameter. Remote Sens. 2019, 11, 451. [CrossRef]

14. Huynen, J.R. Phenomenological Theory of Radar Targets. Ph.D. Thesis, Electrical Engineering, Mathematics and Computer Science, Delft, The Netherlands, 1970.

15. Singha, S.; Ressel, R. Offshore platform sourced pollution monitoring using space-borne fully polarimetric $\mathrm{C}$ and $\mathrm{X}$ band synthetic aperture radar. Mar. Pollut. Bull. 2016, 112, 327-340. [CrossRef]

16. Yang, J.; Peng, Y.N.; Lin, S.M. Similarity between two scattering matrices. Electron. Lett. 2001, 37, 193-194. [CrossRef]

17. Liu, P.; Li, X.; Qu, J.J.; Wang, W.; Zhao, C.; Pichel, W. Oil spill detection with fully polarimetric UAVSAR data. Mar. Pollut. Bull. 2011, 62, 2611-2618. [CrossRef]

18. Schuler, D.L.; Lee, J.S. Mapping ocean surface features using biogenic slick-fields and SAR polarimetric decomposition techniques. IEE Proc. Radar Son. Nav. 2006, 3, 260-270. [CrossRef]

19. Zhu, X.; Li, Y.; Zhang, Q.; Liu, B. Oil film classification using deep learning-based hyperspectral remote sensing technology. ISPRS Int. J. Geo-Inf. 2019, 8, 181. [CrossRef]

20. Wismann, V.; Gade, M.; Alpers, W.; Huhnerfuss, H. Radar signatures of marine mineral oil spills measured by an airborne multi-frequency radar. Int. J. Remote Sens. 1998, 19, 3607-3623. [CrossRef]

21. Alpers, W.; Espedal, H. Oils and surfactants. In Synthetic Aperture Radar Marine User's Manual; National Oceanic and Atmospheric Administration: Washington, DC, USA, 2004.

22. Gade, M.; Alpers, W.; Hühnerfuss, H.; Masuko, H.; Kobayashi, T. Imaging of biogenic and anthropogenic ocean surface films by the multifrequency/multipolarization SIR-C/X-SAR. J. Geophys. Res. Oceans 1998, 103, 18851-18866. [CrossRef]

23. Buono, A.; Nunziata, F.; Migliaccio, M.; Li, X. Polarimetric analysis of compact-polarimetry SAR architectures for sea oil slick observation. IEEE Trans. Geosci. Remote Sens. 2016, 54, 5862-5874. [CrossRef]

24. Tian, W.; Shao, Y.; Yuan, J.; Wang, S.; Liu, Y. An experiment for oil spill recognition using RADARSAT-2 image. In Proceedings of the IEEE International Geoscience and Remote Sensing Symposium (IGARSS), Honolulu, HI, USA, 25-30 July 2010; pp. $2761-2764$.

25. Cloude, S.R.; Pottier, E. An entropy based classification scheme for land applications of polarimetric SAR. IEEE Trans. Geosci. Remote Sens. 1997, 1, 68-78. [CrossRef]

26. Cai, Y.; Zou, Y.; Liang, C.; Zou, B. Research on polarization of oil spill and detection. Acta Oceanologica Sinica 2016, 35, 84-89. [CrossRef]

27. Zou, Y.; Shi, L.; Zhang, S.; Liang, C.; Zeng, T. Oil spill detection by a support vector machine based on polarization decomposition characteristics. Acta Oceanologica Sinica 2016, 35, 86-90. [CrossRef]

28. Espeseth, M.M.; Skrunes, S.; Jones, C.E.; Brekke, C.; Holt, B.; Doulgeris, A.P. Analysis of evolving oil spills in full-polarimetric and hybrid-polarity SAR. IEEE Trans. Geosci. Remote Sens. 2017, 55, 4190-4210. [CrossRef]

29. Migliaccio, M.; Nunziata, F.; Buono, A. SAR polarimetry for sea oil slick observation. Int. J. Remote Sens. 2015, 36, 3243-3273. [CrossRef]

30. Skrunes, S.; Brekke, C.; Jones, C.E.; Espeseth, M.M.; Holt, B. Effect of wind direction and incidence angle on polarimetric SAR observations of slicked and unslicked sea surfaces. Remote Sens. Environ. 2018, 213, 73-91. [CrossRef]

31. Wenguang, W.; Fei, L.; Peng, W.; Jun, W. Oil spill detection from polarimetric SAR image. In Proceedings of the 10th IEEE International Conference on Signal Processing Proceedings, Beijing, China, 24-28 October 2010; pp. 832-835.

32. Breiman, L. Random forests. Mach. Learn. 2001, 45, 5-32. [CrossRef]

33. Dabboor, M.; Howell, S.; Shokr, M.; Yackel, J. The Jeffries-Matusita distance for the case of complex Wishart distribution as a separability criterion for fully polarimetric SAR data. Int. J. Remote Sens. 2014, 35, 6859-6873.

34. Peli, E. Contrast in complex images. J. Opt. Soc. Am. A 1990, 7, 2032-2040. [CrossRef]

35. Song, D.; Zhen, Z.; Wang, B.; Li, X.; Gao, L.; Wang, N.; Xie, T.; Zhang, T. A novel marine oil spillage identification scheme based on convolution neural network feature extraction from fully polarimetric sar imagery. IEEE Access 2020, 8, 59801-59820. [CrossRef]

36. Nunziata, F.; Buono, A.; Migliaccio, M. COSMO—SkyMed synthetic aperture radar data to observe the deep water horizon oil spill. Sustainability 2018, 10, 3599. [CrossRef] 
37. Song, D.; Ding, Y.; Li, X.; Zhang, B.; Xu, M. Ocean oil spill classification with RADARSAT-2 SAR based on an optimized wavelet neural network. Remote Sens. 2017, 9, 799. [CrossRef]

38. Padma, S.; Sanjeevi, S. Jeffries Matusita based mixed-measure for improved spectral matching in hyperspectral image analysis. Int. J. Appl. Earth Obs. Geoinf. 2014, 32, 138-151. [CrossRef]

39. Belgiu, M.; Drăguț, L. Random forest in remote sensing: A review of applications and future directions. ISPRS J. Photogramm. Remote Sens. 2016, 114, 24-31. [CrossRef]

40. Liaw, A.; Wiener, M. Classification and regression by random forest. $R$ News 2002, 2, 18-22.

41. Guanter, L.; Kaufmann, H.; Segl, K.; Foerster, S.; Rogass, K.; Chabrillat, S.; Kuester, T.; Hollstein, A.; Rossner, G.; Chlebek, C.; et al. The EnMAP spaceborne imaging spectroscopy mission for earth observation. Remote Sens. 2015, 7, 8830-8857. [CrossRef]

42. Van der Linden, S.; Rabe, A.; Held, M.; Jakimow, B.; Leitão, P.J.; Okujeni, A.; Schwieder, M.; Suess, S.; Hostert, P. The EnMAP-BoxA toolbox and application programming interface for EnMAP data processing. Remote Sens. 2015, 7, 11249-11266. [CrossRef] 